\title{
The repurposed anthelmintic mebendazole in combination with trametinib suppresses refractory NRASQ61K melanoma
}

\author{
Cynthia M. Simbulan-Rosenthal ${ }^{1, *}$, Sivanesan Dakshanamurthy ${ }^{1,2, *}$, Anirudh Gaur ${ }^{1}$, \\ You-Shin Chen ${ }^{1}$, Hong-Bin Fang ${ }^{3}$, Maryam Abdussamad ${ }^{4}$, Hengbo Zhou ${ }^{4}$, John \\ Zapas $^{4}$, Valerie Calvert ${ }^{5}$, Emanuel F. Petricoin ${ }^{5}$, Michael B. Atkins ${ }^{2}$, Stephen W. \\ Byers $^{1,2}$ and Dean S. Rosenthal ${ }^{1}$ \\ ${ }^{1}$ Department of Biochemistry and Molecular \& Cellular Biology, Georgetown University Medical Center, Washington, DC, USA \\ 2 Department of Oncology, Lombardi Comprehensive Cancer Center, Georgetown University Medical Center, Washington, \\ DC, USA \\ ${ }^{3}$ Department of Biostatistics, Bioinformatics, and Biomathematics, Georgetown University Medical Center, Washington, DC, \\ USA \\ ${ }^{4}$ MedStar Franklin Square Medical Center, Baltimore, MD, USA \\ ${ }^{5}$ Center for Applied Proteomics and Molecular Medicine, George Mason University, Manassas, VA, USA \\ * These authors are co-first authors \\ Correspondence to: Dean S. Rosenthal, email: rosenthd@georgetown.edu \\ Keywords: mebendazole, drug repurposing, melanoma, ERK pathway, BRAF \\ Received: March 31, $2016 \quad$ Accepted: October 13, $2016 \quad$ Published: February 02, 2017
}

\section{ABSTRACT}

Structure-based drug repositioning in addition to random chemical screening is now a viable route to rapid drug development. Proteochemometric computational methods coupled with kinase assays showed that mebendazole (MBZ) binds and inhibits kinases important in cancer, especially both BRAFWT and BRAF ${ }^{\mathrm{V} 600 E}$. We find that MBZ synergizes with the MEK inhibitor trametinib to inhibit growth of BRAFWTNRAS ${ }^{61 K}$ melanoma cells in culture and in xenografts, and markedly decreased MEK and ERK phosphorylation. Reverse Phase Protein Array (RPPA) and immunoblot analyses show that both trametinib and MBZ inhibit the MAPK pathway, and cluster analysis revealed a protein cluster showing strong $M B Z+$ trametinib - inhibited phosphorylation of MEK and ERK within 10 minutes, and its direct and indirect downstream targets related to stress response and translation, including EIK1 and RSKs within 30 minutes. Downstream ERK targets for cell cycle, including CMYC, were down-regulated, consistent with $S$ - phase suppression by MBZ+trametinib, while apoptosis markers, including cleaved caspase-3, cleaved PARP and a sub-G1 population, were all increased with time. These data suggest that MBZ, a welltolerated off-patent approved drug, should be considered as a therapeutic option in combination with trametinib, for patients with NRAS ${ }^{961 m u t}$ or other non-V600E BRAF mutant melanomas.

\section{INTRODUCTION}

The paradigm of targeted therapy, the one-drug one-target disease approach, has issues including the development of resistance and, thus, there is a need for new therapeutics to target newly amplified or mutated proteins [1]. As cancer progresses by multiple pathways, targeting one pathway alone is usually insufficient. Consequently, combination targeted therapies have been advocated as a new approach to cancer treatment using either multi- target inhibitors or combinations of single-target agents. Thus, promiscuous inhibitors such as sorafenib and sunitinib are effective at disrupting multiple nodes in cellsignaling pathways. However, since these agents may have unacceptable side effects, dose titration is problematic [2]. The latest technologies used for new drug discovery are in part intended to circumvent these challenges, and while the number of new molecular entities introduced in 2014 increased, trends in drug development have been variable [3]. An alternative approach is to utilize available drugs 
and repurpose them for other indications.

Mebendazole (MBZ; methyl N-[6-(benzoyl)-1Hbenzimidazol-2-yl] carbamate), an inexpensive chewable anthelmintic drug, has been widely used since the early 1970s [4] and is non-toxic even when administered in high doses [5]. MBZ acts at the colchicine-binding site of roundworm tubulin, and disrupts its polymerization [6, 7]. MBZ does not cause side effects characteristic of other anti-microtubule drugs, including taxanes and the vinca alkaloids [8].

The microtubule-disrupting properties of MBZ and other benzimidazole carbamates such as albendazole stimulated interest in these drugs as anti-cancer agents. MBZ inhibits mitotic spindles, induces G2/M arrest and apoptosis in human lung cancer cells, and suppresses their ability to form tumors in nude mice without host toxicity $[9,10]$. However, the affinity of MBZ for human tubulin is much less than that of roundworm tubulin and it is unlikely that circulating MBZ levels would ever reach levels sufficient to block human tubulin in vivo. In addition to targeting tubulin, studies by our lab and others revealed that MBZ inhibits VEGFR-2, PDGFRA and PDGFRB at $3600 \mathrm{nM}, 820 \mathrm{nM}$ and $660 \mathrm{nM}$, respectively [11-13]. MBZ inhibits growth of melanoma cell lines in vitro and in vivo, accompanied by changes in tubulin polymerization, BCL2 phosphorylation and apoptosis [14]. While two clinical trials of MBZ for glioma are currently ongoing, two case reports for patients with either metastatic adrenocortical cancer or metastatic colon cancer highlighted evidence of clinical benefit. In the former case, some regression in metastatic lesions were observed, and the cancer remained stable on MBZ monotherapy, with the patient tolerating treatment without side effects until progression at 24 months [15]. In the latter case, near complete regression of metastatic lesions in lungs and lymph nodes and partial regression in the liver was observed [16]. A further five patients were treated with MBZ, including one experiencing a minor tumor response, in a reported personal communication [17].

Current cancer therapies have focused on targeting driver mutations, including oncogenic BRAF and NRAS, which are frequent in melanomas. BRAF ${ }^{\mathrm{V} 600 \mathrm{E}}$ and $\mathrm{BRAF}^{\mathrm{V} 600 \mathrm{~K}}$ are found in $46 \%$ and $9 \%$ of melanomas, respectively. Additionally, $10 \%$ of melanomas previously classified as "BRAF" ${ }^{\mathrm{WT}}$ " tumors actually harbor non$\mathrm{V} 600 \mathrm{E} / \mathrm{K}$ mutations in BRAF. In fact, more than 30 mutations of the $B R A F$ gene associated with human cancers have been identified [18], many of which may be sensitive to trametinib since these show deregulated stimulation of MEK1/2. Acquired resistance to the targeted therapeutics dabrafenib (GSK 2118436a; a BRAF ${ }^{\mathrm{V} 600 \mathrm{E} / \mathrm{K}}$ inhibitor) and/or trametinib (GSK1120212; a MEK1/2 inhibitor) is associated with development of additional mutations, including those activating NRAS. Patients with melanomas harboring NRAS ${ }^{\text {mut }} / \mathrm{BRAF}^{\mathrm{WT}}$ signatures ( $\sim 21 \%$ of patients) have limited treatment options and are refractory to current targeted therapies.

In the current study, we report that the combination of MBZ and trametinib suppresses proliferation of patient-derived melanoma cell lines harboring NRAS ${ }^{\text {mut }}$ $\mathrm{BRAF}^{\mathrm{WT}}$ as determined by gene sequencing, and significantly attenuates their growth in xenografts in immunocompromised mice. Reverse phase protein array (RPPA) based protein pathway activation mapping and immunoblot analyses revealed specific inhibition of the MAPK pathway and downstream regulation by MBZ or trametinib alone or in combination, within 10 minutes of drug treatment. At later time points, MBZ+trametinib induces markers of apoptosis, including proteolytic activation of caspase-3 and PARP cleavage, increased caspase activity as measured by fluorometric assays and increased levels of apoptotic sub-G1 cells. A reduction of cells in $\mathrm{S}$ phase was also observed in cells exposed to trametinib (by $24 \mathrm{~h}$ ) or trametinib+MBZ (by $8 \mathrm{~h}$ ), concurrent with an increase in G2 by $24 \mathrm{~h}$ and in G1 by $48 \mathrm{~h}$. Thus, these results are consistent with the suppression of the MEK1/2 by trametinib and suppression of BRAF ${ }^{\mathrm{WT}}$ by MBZ, leading to the combinatorial rapid shutoff of ERK activity, as well as downstream targets of ERK. MBZ is therefore a viable nontoxic option that can be used to increase the effectiveness of trametinib in NRAS ${ }^{\text {mut/ }}$ $\mathrm{BRAF}^{\mathrm{WT}}$ melanoma.

\section{RESULTS}

\section{In silico repurposing technology and in vitro kinase assays show that MBZ inhibits mutant and wild-type BRAF}

Efforts to develop drugs targeting mutant BRAF led to FDA approval of vemurafenib in 2011 and dabrafenib in 2013. While these drugs either used alone or particularly when used in combination with MEK inhibitors such as trametinib or cobimetinib have been extremely successful at shrinking tumors, delaying disease progression and prolonging survival, resistance to them commonly develops at a median of 7-12 months typically through the selection of variants exhibiting mutations in other kinase pathway members, most notably NRAS. Our refined TMFS method [12] identified MBZ as a hit with a mode of inhibition that binds both wild type and V600E mutant BRAF (Figure $1 A, 1 B$ ). In addition, other MAPK pathway proteins including CRAF and MEK were identified. Our in-vitro assays confirmed that $\mathrm{BRAF}$ and $\mathrm{MEK}$ were inhibited by $\mathrm{MBZ}$ in the $\mathrm{nM}$ range (Figure $1 C$ ), with $\mathrm{MBZ}$ inhibiting both BRAF ${ }^{\mathrm{V} 600 \mathrm{E}}$ and $\mathrm{BRAF}^{\mathrm{WT}}$ with a $\mathrm{K}_{\mathrm{d}}$ of 210 and $230 \mathrm{nM}$, respectively, in agreement with previous results with a kinase screen of MBZ, chosen for its ability to inhibit colon cancer growth [13].

Both sorafenib, a pan-kinase inhibitor that interacts 
with BRAF [18], and RAF265, a RAF/VEGFR dual kinase inhibitor [19], bind to the DFG-out (indicating the positions of the three key amino acids aspartate, phenylalanine, and glycine) inactive conformation of $\mathrm{BRAF}^{\mathrm{WT}}$ and BRAF ${ }^{\mathrm{V} 600 \mathrm{E}}$ at the ATP binding site. In contrast, vemurafenib [20] and dabrafenib [21] bind to the DFG-in active conformation of the ATP binding site. These active conformation inhibitors are highly BRAFselective compared to other kinases [20]. Our structurebased modeling shows that MBZ binds both inactive and active conformations of BRAF (Figure $1 A, 1 B$ ). The BRAF structural model revealed that MBZ occupies the
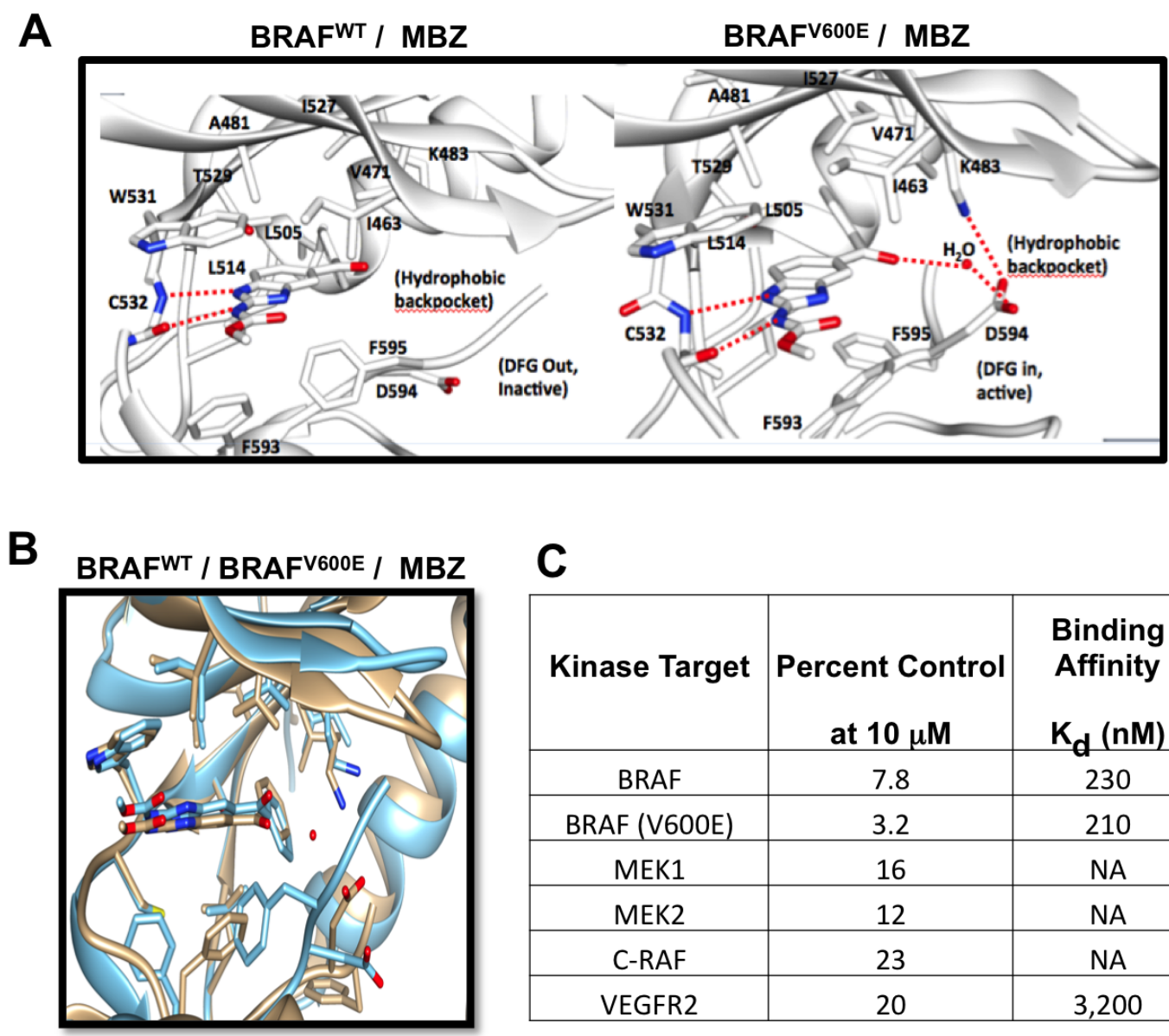

C
\begin{tabular}{|c|c|c|}
\hline Kinase Target & Percent Control & $\begin{array}{c}\text { Binding } \\
\text { Affinity } \\
\text { at } 10 \mu \mathbf{M}\end{array}$ \\
$\mathbf{K}_{\mathbf{d}}$ (nM) \\
\hline BRAF & 7.8 & 230 \\
\hline BRAF (V600E) & 3.2 & 210 \\
\hline MEK1 & 16 & NA \\
\hline MEK2 & 12 & NA \\
\hline C-RAF & 23 & NA \\
\hline VEGFR2 & 20 & 3,200 \\
\hline
\end{tabular}
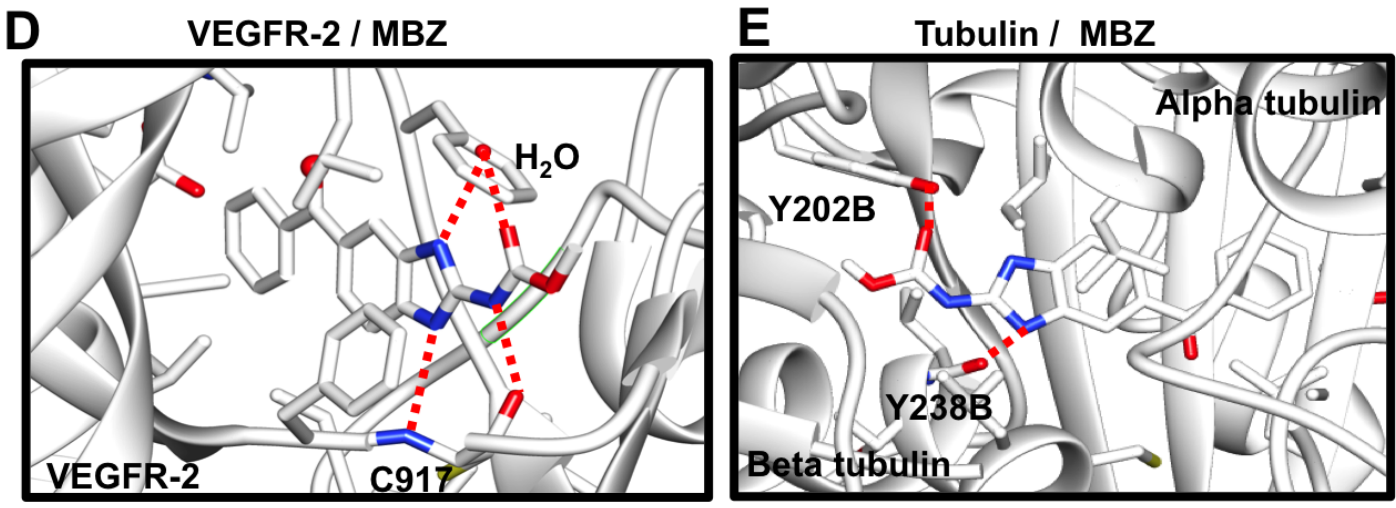

Figure 1: Structure of $\mathrm{MBZ}$ with $\mathrm{BRAF}^{\mathrm{WT}}$ (left) or $\mathrm{BRAF}^{\mathrm{V} 600 \mathrm{E}}$ (right) are displayed showing residues critical for binding; hydrogen bonds are shown as dotted red lines. A. Structure overlay of BRAFWT (blue) and BRAF ${ }^{\mathrm{V} 600 \mathrm{E}}$ (tan) with MBZ B. MBZ inhibition of kinase activity and binding affinities for different targets C. Structure model of VEGFR-2 (PDB:2OH4; D.) and Tubulin (PDB:3N2G; E.) with MBZ. Critical binding site residues are displayed; hydrogen bonds shown as dotted red lines and water molecule shown as ball model. 
ATP-binding site and stabilizes both the active DFG-in and inactive DFG-out conformations. MBZ is surrounded by residues I463, V471, A481, K483, L505, L514, I527, T529, W531, C532, D594, and F595, and its binding is driven by hydrophobic and hydrogen bond interactions at the ATP site. An amide proton at the 2-position and a nitrogen atom at the $\mathrm{N}-1$ position of the methyl $\mathrm{N}-(1 \mathrm{H}-$ benzimidazol-2-yl)carbamate moiety of MBZ form a significant hydrogen bond interaction with the backbone $\mathrm{C}=\mathrm{O}$ and $-\mathrm{NH}$ of $\mathrm{C} 532$ in the kinase hinge regions of both the DFG-in and the DFG-out forms of BRAF ${ }^{\mathrm{WT}}$ and $\mathrm{BRAF}^{\mathrm{V} 600 \mathrm{E}}$. The methyl group connected to amide moiety of methyl N-(1H-benzimidazol-2-yl)carbamate forms a hydrophobic interaction with the indole ring of W531, and is suitably placed, whereas larger hydrophobic replacements would create steric hindrance due to space constraints in the binding site between the indole side chain of W531 and G534. Two interactions between MBZ and BRAF ${ }^{\mathrm{V} 600 \mathrm{E}}$, but not BRAF ${ }^{\mathrm{WT}}$ include: 1) an additional hydrophobic interaction between F593 and the benzimidazole moiety, and 2) a water-mediated hydrogen bond interaction between D594 and a keto group of the methyl N-(1H-benzimidazol-2-yl)carbamate moiety. These differences explain the slightly higher $\mathrm{K}_{\mathrm{d}}$ value observed for $\mathrm{BRAF}^{\mathrm{WT}}$. MBZ does not interact with the BRAF lipophilic back pocket, unlike other BRAF inhibitors, lowering its affinity for C-RAF.

TMFS analysis also reveals that MBZ interacts with VEGFR2 (Figure 1D), consistent with its structural similarity to the benzimidazole-urea VEGFR2 inhibitor (PDB:2OH). However, MBZ showed more potency towards BRAF (Figure 1C) than to VEGFR2 [12], probably due to the absence of its interaction with residues lining the ATP site back pocket, which is more important for VEGFR2 than for BRAF ${ }^{\mathrm{V} 600 \mathrm{E}}$. In addition, the F918 phenyl ring of VEGFR2 restricts the diversified and nonplanar conformation of MBZ, compared to the W531 indole ring of BRAF. Consistent with previous studies [14], MBZ also interacts with tubulin (Figure $1 E$ ).

\section{The combination of $\mathrm{MBZ}$ and trametinib is cytotoxic to NRAS- and BRAF-mutant melanoma cells}

Based on the ability of MBZ to target both mutant and wild-type BRAF, two patient-derived melanoma cell lines (BAK and BUL) harboring the same BRAFWT/ NRAS ${ }^{\mathrm{Q} 61 \mathrm{~K}}$ mutation profile and another melanoma cell line (STU) with a BRAF ${ }^{\mathrm{V} 600 \mathrm{~K}} / \mathrm{NRAS}^{\mathrm{WT}}$ mutation signature were exposed for $72 \mathrm{~h}$ to increasing concentrations of dabrafenib (D), trametinib (T), MBZ, or combinations of $\mathrm{T}+\mathrm{D}$ or $\mathrm{T}+\mathrm{MBZ}$. XTT cell viability assays revealed that, while all three cell lines exhibited resistance to dabrafenib except at the highest doses tested, MBZ showed weak cytotoxic activity as a single agent, but synergized strongly with trametinib in both BAK and BUL cells, but was either antagonistic (at low concentrations) or additive (at high concentrations) in STU cells. T and D were also synergistic in BAK and BUL, but not STU, although maximum inhibition was greater in $\mathrm{T}+\mathrm{MBZ}$ - treated cells (Figure 2; Supplementary Table 2, Supplementary Figure 1). Consequently, the MBZ+trametinib combination may represent a potential therapy in NRAS mutant melanoma cells.

To determine whether the reduced cell numbers were due to inhibition of proliferation or increased cell death, apoptosis and cell cycle assays were performed in BAK and BUL melanoma cells. Caspase-3 activity (Figure $3 A, 3 F$ ), as well as a sub-G1 population (Figure $3 B, 3 G$ ) was induced earlier, and to a greater extent in cells exposed to $\mathrm{T}+\mathrm{MBZ}$ than to either drug alone, indicating that this combination rapidly and robustly induces apoptosis. In both BAK and BUL cells, trametinib and/or the combination of $\mathrm{T}+\mathrm{MBZ}$ also decreased the percentage of cells in $\mathrm{S}$ phase of the cell cycle at all time points, with concomitant increases in G2 and G1 phases of the cell cycle by $8 \mathrm{~h}$ or $24 \mathrm{~h}$, respectively (Figure $3 C-3 E, 3 H-3 J$ ).

\section{The combination of $\mathrm{MBZ}$ and trametinib reduces tumor growth in xenografts}

To determine if MBZ and trametinib are effective against $\mathrm{BRAF}^{\mathrm{WT}} / \mathrm{NRAS}^{\mathrm{Q} 61 \mathrm{~K}}$ melanoma in vivo, BAK cells were xenografted into nude mice, and treated with MBZ, trametinib, or their combination. Two different doses of trametinib were administered to different groups of mice daily by gavage $(0.1 \mathrm{mg} / \mathrm{kg} \mathrm{LDT}$, or $3 \mathrm{mg} / \mathrm{kg}$ HDT). A third group of mice was treated with a dose of MBZ similar to that used for helminthic infections $(40 \mathrm{mg}$ / $\mathrm{kg}$ on alternate days), while a fourth and fifth group of mice received a combination of MBZ and either LDT or HDT. These trametinib doses bracket those prescribed for patients (from $1 / 4$ - to 7-fold), and our MBZ doses are much less than those safely used in patients based on dose per body surface area (BSA; Materials and Methods), and are similar to those in previous preclinical studies [22, 23]. The vehicle served as a control for the sixth group of mice.

While trametinib as a single agent did not show any significant tumor-suppressive effects (HDT vs. control $p=$ 0.26 ; LDT $v s$. control $p=0.65$, Supplementary Table 1), tumor growth was significantly inhibited in mice treated with MBZ in combination with either high (HDT+MBZ $v s$. vehicle $p=.038$ ) or low (LDT + MBZ vs. vehicle $p=$ .066) trametinib doses, although not quite to a significant level in the latter case, without loss in weight or any other obvious adverse effects (Figure 4A, 4B). Remarkably, the HDT $+\mathrm{MBZ}$ combination group remained alive long after the other arms had been euthanized due to the size of the NRAS ${ }^{\mathrm{Q} 61 \mathrm{~K}}$ melanoma xenografts at 42 days. Tumors from xenografts collected at the termination of the 
A
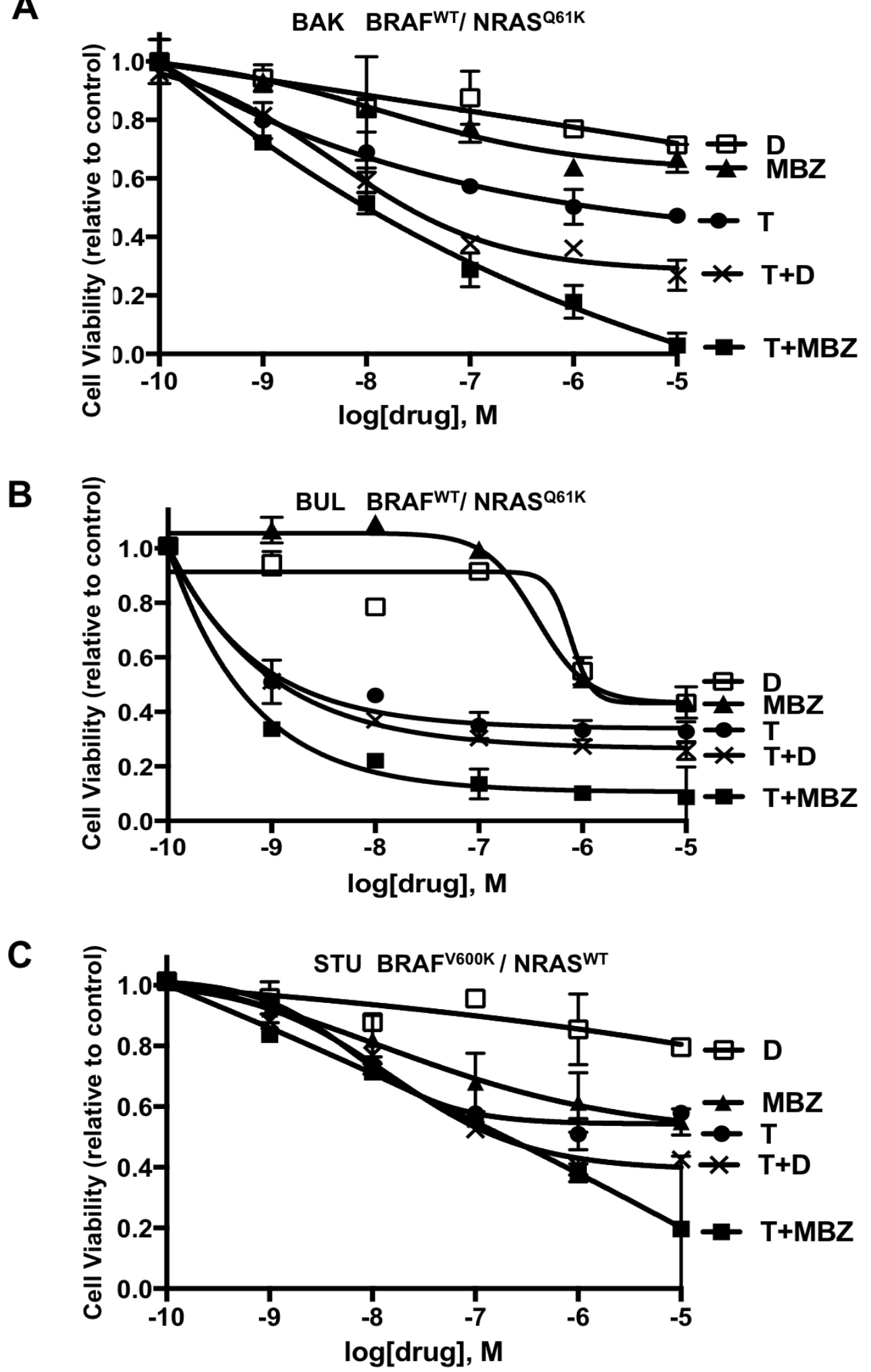

Figure 2: Decreased viability of melanoma cells exposed to MBZ, dabrafenib (D), trametinib (T), or combinations of T+D or MBZ+trametinib. A. BAK $\left(\mathrm{BRAF}^{\mathrm{WT}} / \mathrm{NRAS}^{\mathrm{Q} 61 \mathrm{~K}}\right)$, B. BUL (BRAF $\left.{ }^{\mathrm{WT}} / \mathrm{NRAS}^{\mathrm{Q} 61 \mathrm{~K}}\right)$ and $\mathbf{C}$. STU $\left(\mathrm{BRAF}^{\mathrm{V} 600 \mathrm{~K}} / \mathrm{NRAS}^{\mathrm{WT}}\right) \mathrm{melanoma}^{\mathrm{T}}$ cells were exposed to the indicated concentrations of MBZ, D, T, T+D or MBZ+trametinib (T+MBZ) for $72 \mathrm{~h}$, and subjected to XTT cell viability assays, as described in Materials and Methods. Error bars represent mean \pm SD for triplicates. Significant growth inhibition was observed at $\geq 1 \mathrm{nM}$ for $\mathrm{T}$ or $\mathrm{T}+\mathrm{MBZ}, \geq 10 \mathrm{nM}$ for $\mathrm{MBZ}$ alone, and $\geq 1 \mu \mathrm{M}$ for $\mathrm{D}$ (2-way ANOVA). The results shown are based on a single experiment in triplicate, and repeated in three independent experiments with essentially the same results. 
experiment were then subjected to immunoblot analysis to determine the protein levels and phosphorylation status of components of the MAPK pathway in vivo. Whereas MBZ and trametinib alone each demonstrate the ability to suppress MEK and ERK phosphorylation, only the combination of HDT+MBZ completely abrogated both MEK1/2 and ERK1/2 phosphorylation (Figure 4C, 4D).
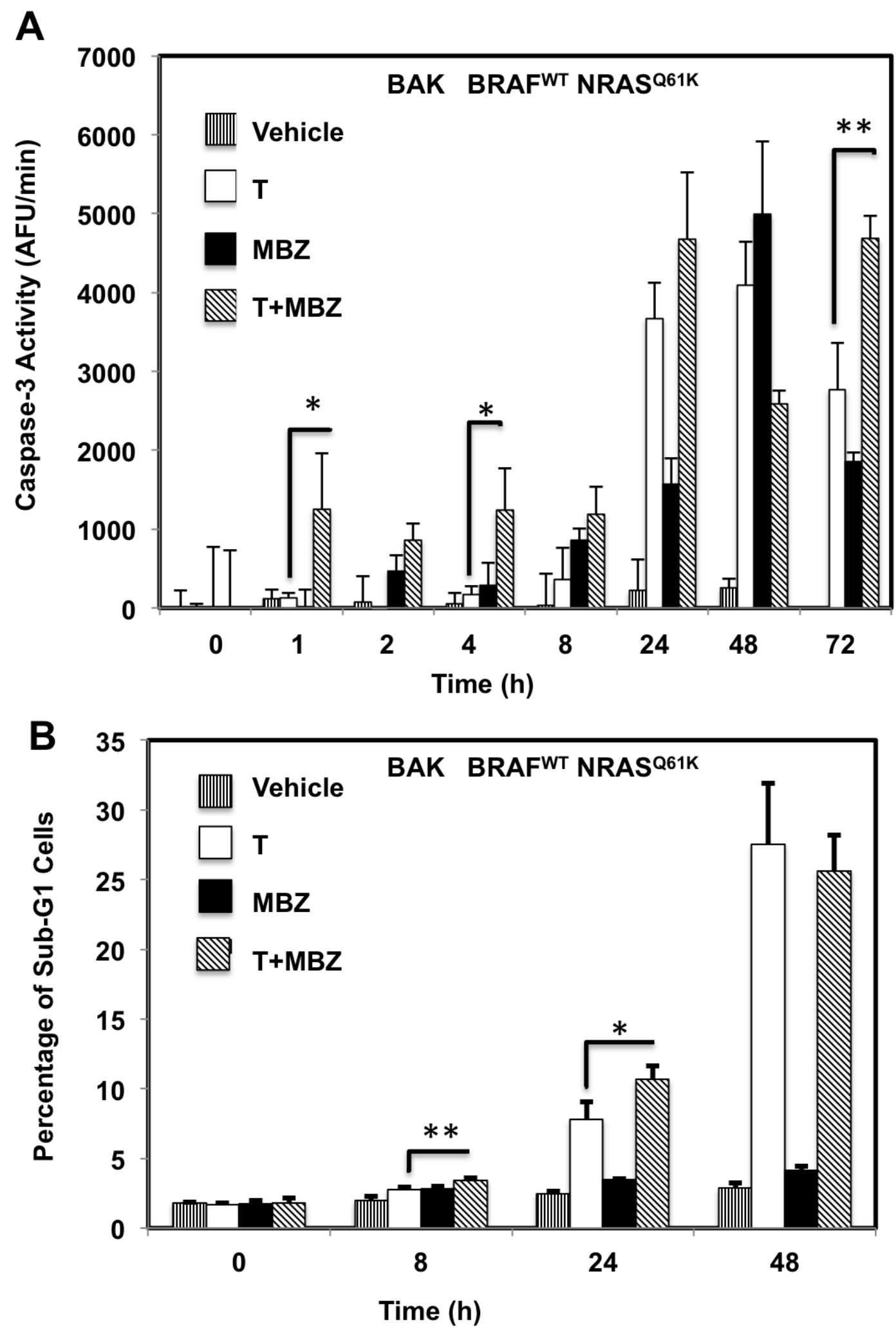

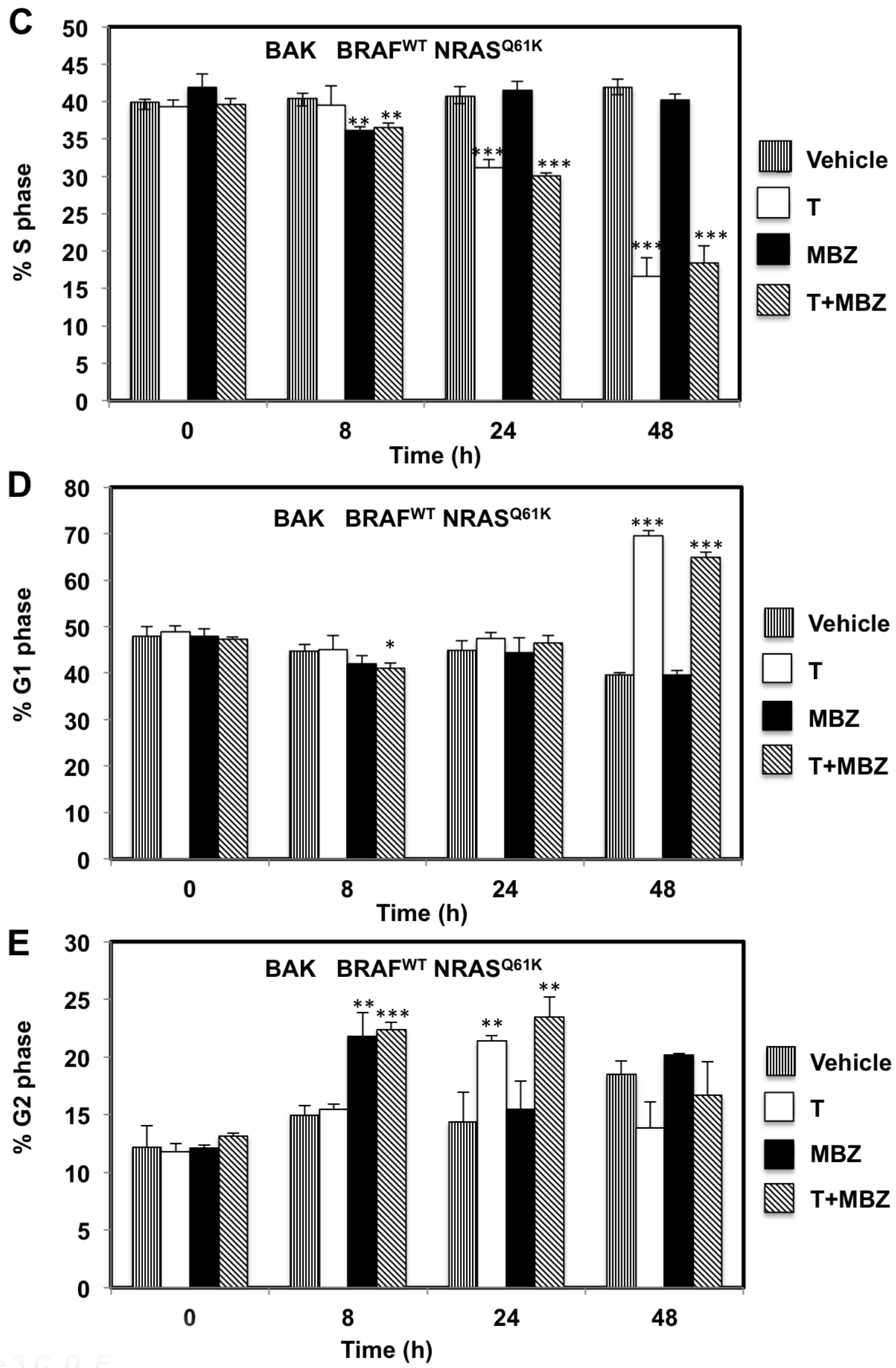
F
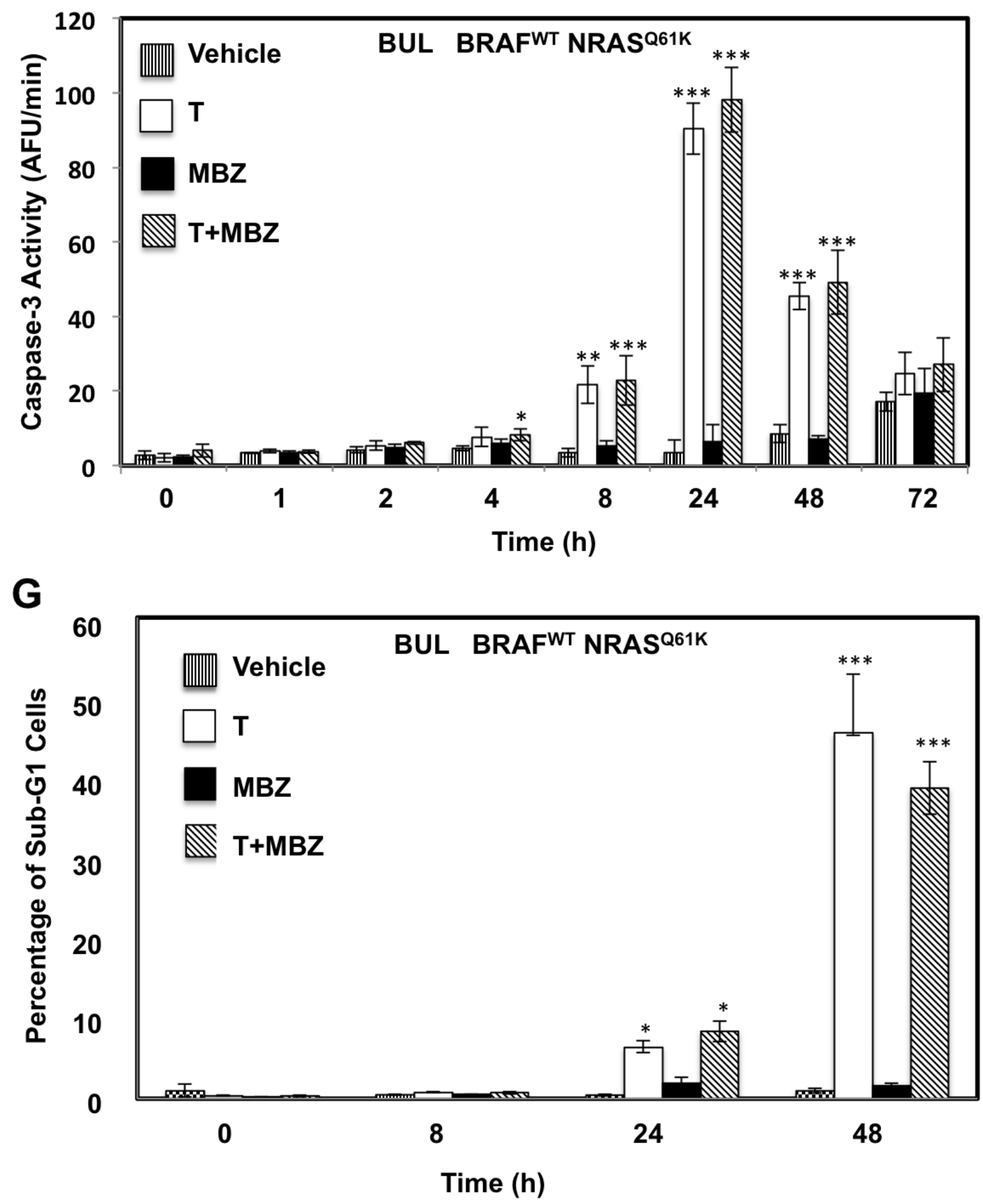

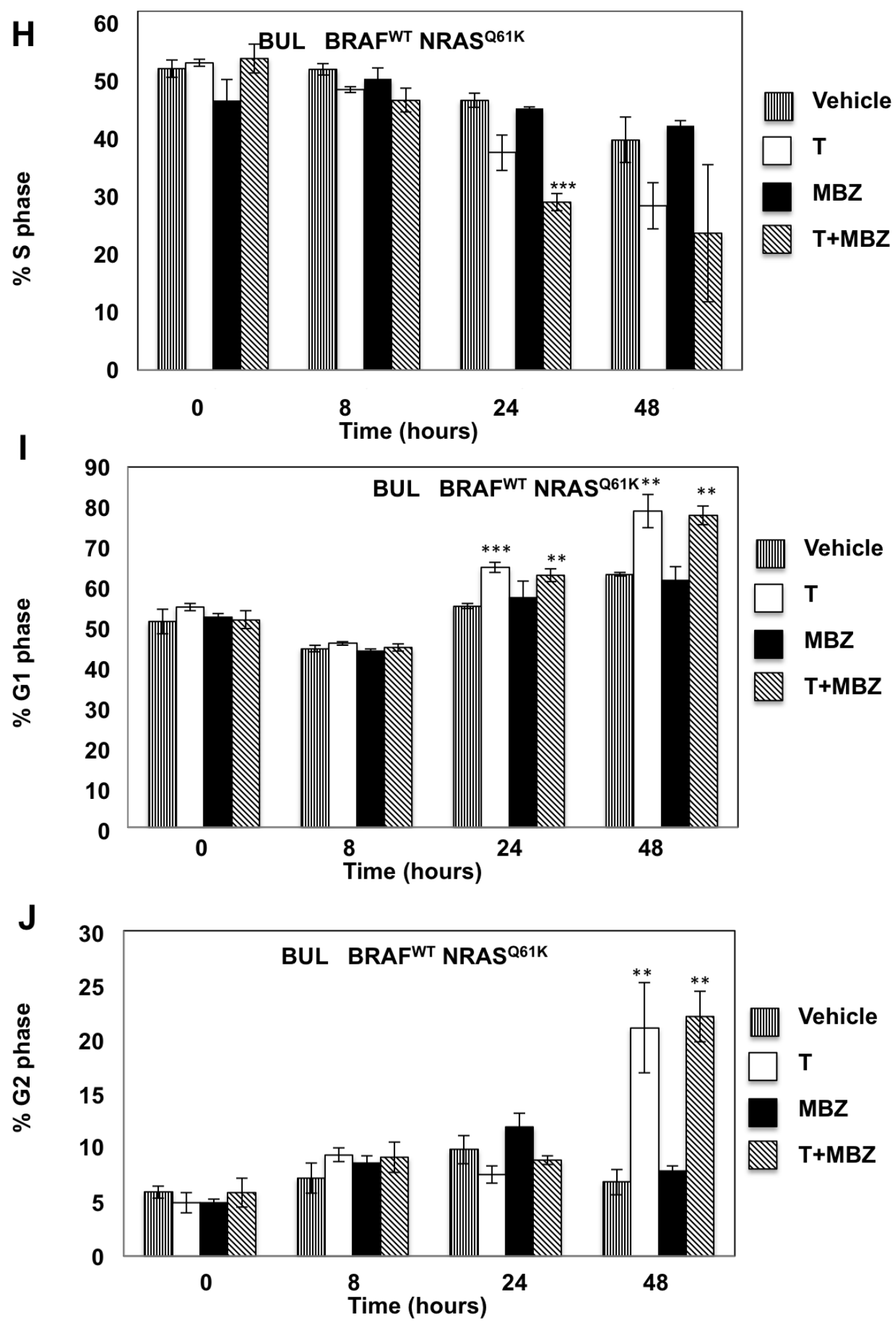

Figure 3: MBZ+trametinib induces apoptosis and decreases the percentage of melanoma cells in $S$ phase of the cell cycle. BAK A.-E. or BUL F.- J. melanoma cells were exposed to $100 \mathrm{nM}$ of MBZ, trametinib, or a combination of the two (T+MBZ); cytosolic extracts were derived at indicated times and subjected to fluorometric analysis using DEVD-AMC as a substrate (A, F), or cells were fixed in EtOH, stained with PI, and subjected to FACS analysis to determine the number of cells in sub-G1 (B, G), S phase (C, H), G1 phase (D, I), or G2 phase of the cell cycle (E, J). Results are the means \pm S.D. of three replicates of a representative experiment. Statistical analysis of T versus T+MBZ groups (A, B) and between vehicle and treatment groups (C-J); 1, 2, or 3 asterisks $(*)$ represent $p<0.05, p<$ 0.001 , and $p<0.0001$, respectively. 
MBZ and trametinib target the MEK/ERK pathway

Given the potential changes in tumor cell signaling and survival over the long time course of the xenografts, we examined potential mechanisms by which MBZ+trametinib exerts its cytotoxic effects using cultured BAK NRAS ${ }^{\text {Q61K }}$ melanoma cells. Cells were exposed to MBZ (10 nM or $100 \mathrm{nM})$, trametinib (10 nM or $100 \mathrm{nM})$, or a combination of the two for 1,8 , or $24 \mathrm{~h}$, after which cell extracts were subjected to reverse-phase protein array (RPPA) analysis. Unsupervised hierarchal clustering of rows revealed that the phosphorylation of a number of proteins associated with the MEK/ERK pathway was down-regulated by MBZ, trametinib, or their combination, although the response to $\mathrm{MBZ}+$ trametinib $(\mathrm{T}+\mathrm{M})$ was more rapid and robust (Figure $5 \mathrm{~A}$ ). Thus, phosphorylation of ERK and its downstream targets involved in translation, including p90RSK, ribosomal protein S6, and eIF4E were all concomitantly inhibited within $1 \mathrm{~h}$ of drug exposure,

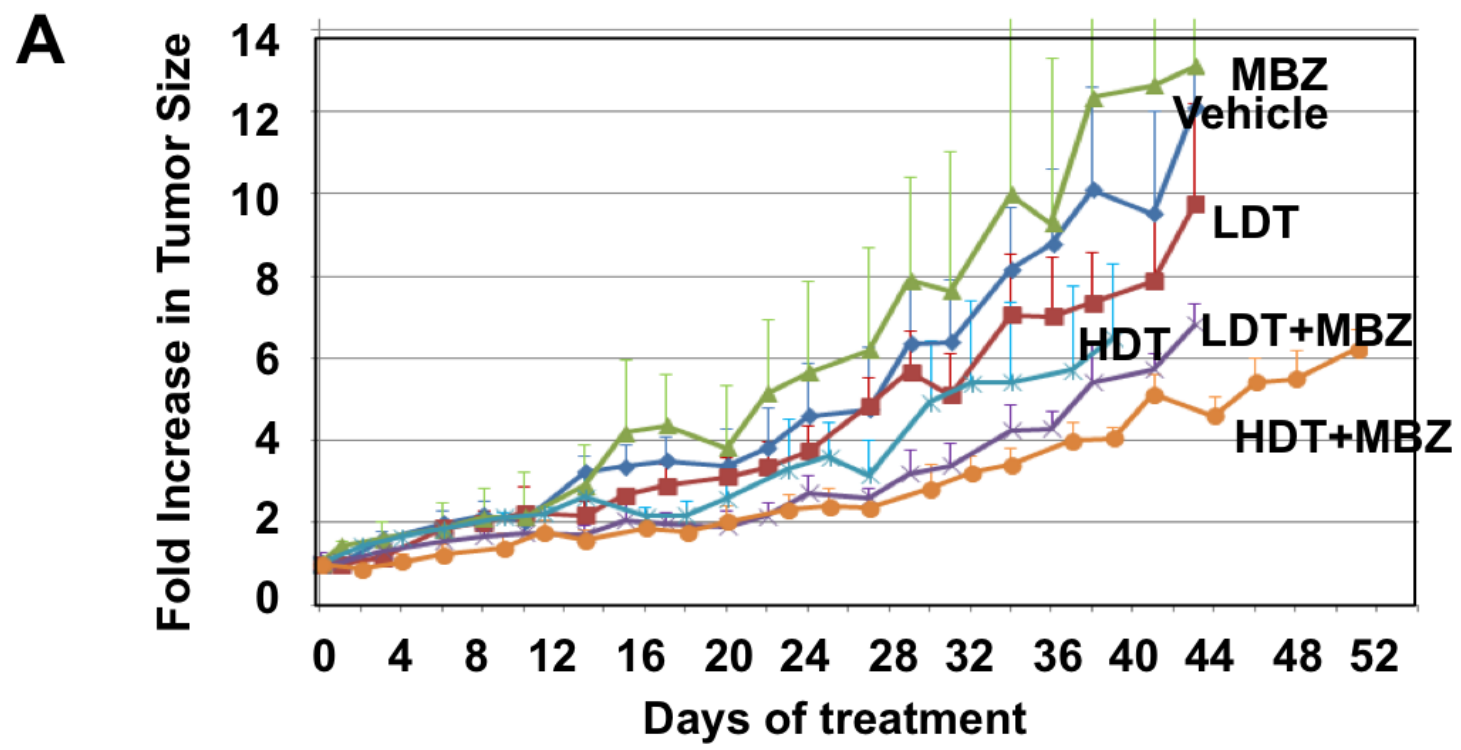

B

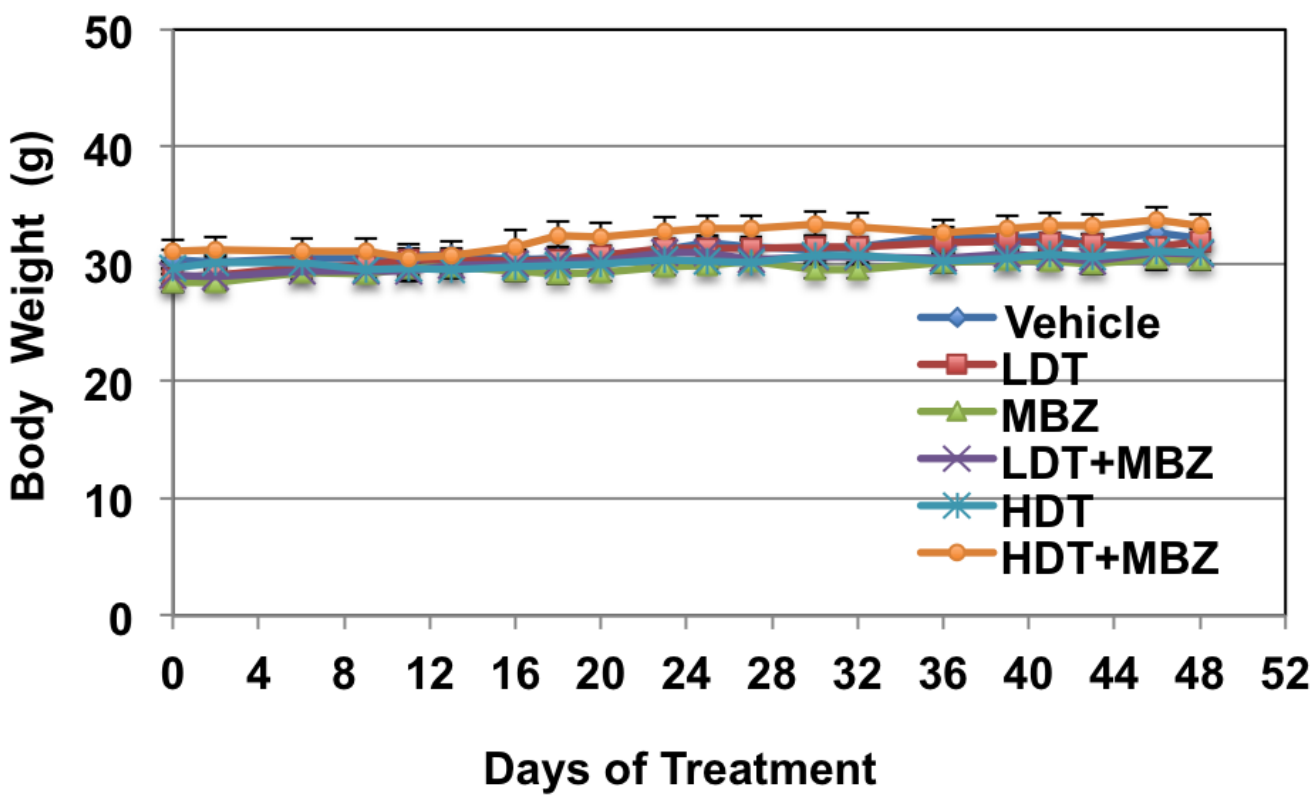



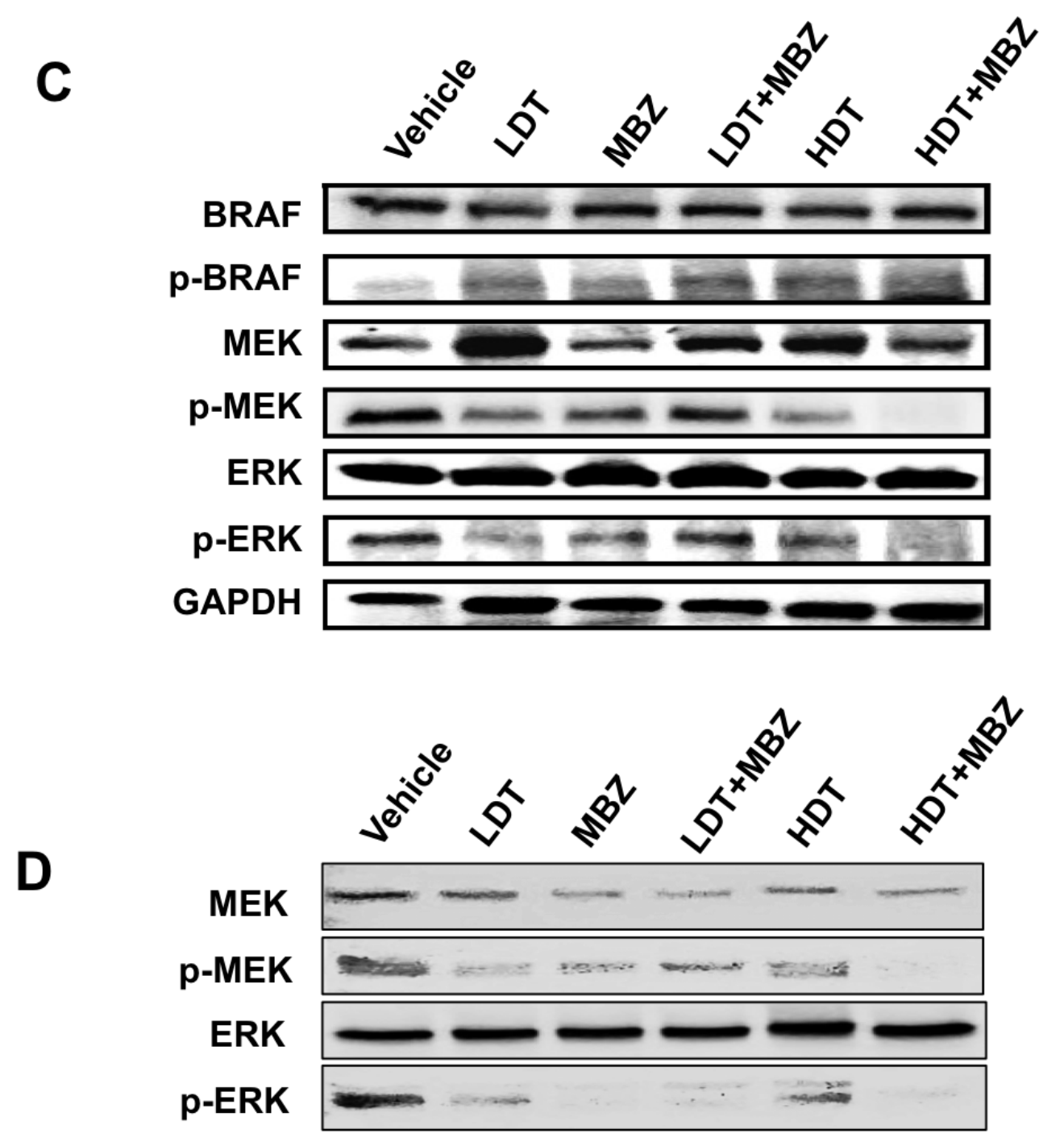

Figure 4: MBZ+trametinib significantly inhibit tumor growth and phosphorylation of MEK and ERK in vivo. Athymic mice were injected with $3 \times 10^{6} \mathrm{BAK}$ melanoma cells, and tumors were allowed to grow to a volume of $100 \mathrm{~mm}^{3}$. Animals were then gavaged with vehicle emulsion control, $40 \mathrm{mg} / \mathrm{kg} / \mathrm{qad} \mathrm{MBZ}$, low dose trametinib (LDT; $0.1 \mathrm{mg} / \mathrm{kg} / \mathrm{qd}$ ), high dose trametinib (HDT; 3 $\mathrm{mg} / \mathrm{kg} / \mathrm{qd}$ ) or a combination of MBZ and LDT or HDT. A. Tumor widths and lengths were measured and volumes calculated as $w^{2} x l / 2$, where width is defined as the smaller of the tumor dimensions. Time 0 is the tumor volume on the first day of treatment; tumor sizes were normalized to their size at time 0 of drug treatment. B. Mice were weighed every other day and body weights (g) plotted over time. Data from four experiments was combined for statistical analysis, to compare every mouse from each treatment group. The total mice for all experiments included vehicle control $(n=13)$, LDT $(n=12)$, MBZ $(n=12)$, HDT $(n=12)$, LDT $+\operatorname{MBZ}(n=12)$, and HDT+MBZ $(n=$ $15)$. The results are shown as the mean $( \pm \mathrm{SD})$ of tumor volume in each group. C., D. ERK and MEK phosphorylation is suppressed in large (C) or small (D) tumor xenografts from mice treated with $\mathrm{T}+\mathrm{MBZ}$. Tumor extracts were derived from xenografts, then subjected to immunoblot analysis using antibodies specific for total BRAF, phospho-BRAF, ERK1/2, phospho-ERK1/2, total MEK1/2, phosphoMEK1/2, or GAPDH as a loading control.

and remained hypophosphorylated for $24 \mathrm{~h}$ in the $\mathrm{T}+\mathrm{M}$ groups; hypophosphorylated ELK1 S383 (a known ERK kinase substrate) was also associated with this cluster (Figure 5A; Supplementary Figure 2A). Levels of LC3B and Beclin-1, key regulatory proteins that control autophagy, and known ERK pathway substrates, were also reduced in this cluster [24, 25]. Proteins characteristic of apoptosis were elevated with time, including the proteolytically activated form of caspase- 3 and cleaved PARP, while total levels of the cell cycle progression protein $\mathrm{cMYC}$ were reduced (Figure $5 A$; Supplementary Figure 2B), consistent with the effects of $\mathrm{MBZ}$ and 
trametinib on these two processes (Figure 3).

The potential pathway for early suppression of phospho-ERK and its targets, leading to apoptosis and cell cycle suppression was next examined by immunoblot analysis. Remarkably, whereas the pMEK1/2 S217/221 activating phosphorylation was not inhibited by trametinib or MBZ alone, their combination completely abolished detectable MEK phosphorylation within $30 \mathrm{~min}$ (Figure $5 B$ ). While levels of total ERK $1 / 2$ remained constant throughout the time course for all treatment groups, the activating phosphorylation of ERK1/2 (T202/Y204) was diminished by MBZ or trametinib alone. However, the combination of $\mathrm{MBZ}+$ trametinib completely abrogated ERK phosphorylation, such that phospho-ERK was undetectable by $30 \mathrm{~min}$ of treatment (Figure $5 \mathrm{C}$ ). Consistent with the regulation of BCL2 levels by MEK/ ERK [26], BCL2, but not BCLXL levels, were slightly reduced by $\mathrm{MBZ}+$ trametinib by $24 \mathrm{~h}$ (Figure $5 D$ ). Further, cells treated with the $\mathrm{MBZ}+$ trametinib combination exhibited marked suppression of the inactivating phosphorylation of the pro-apoptotic ERK substrate BAD S11 [27], coincident with a time-dependent increase in

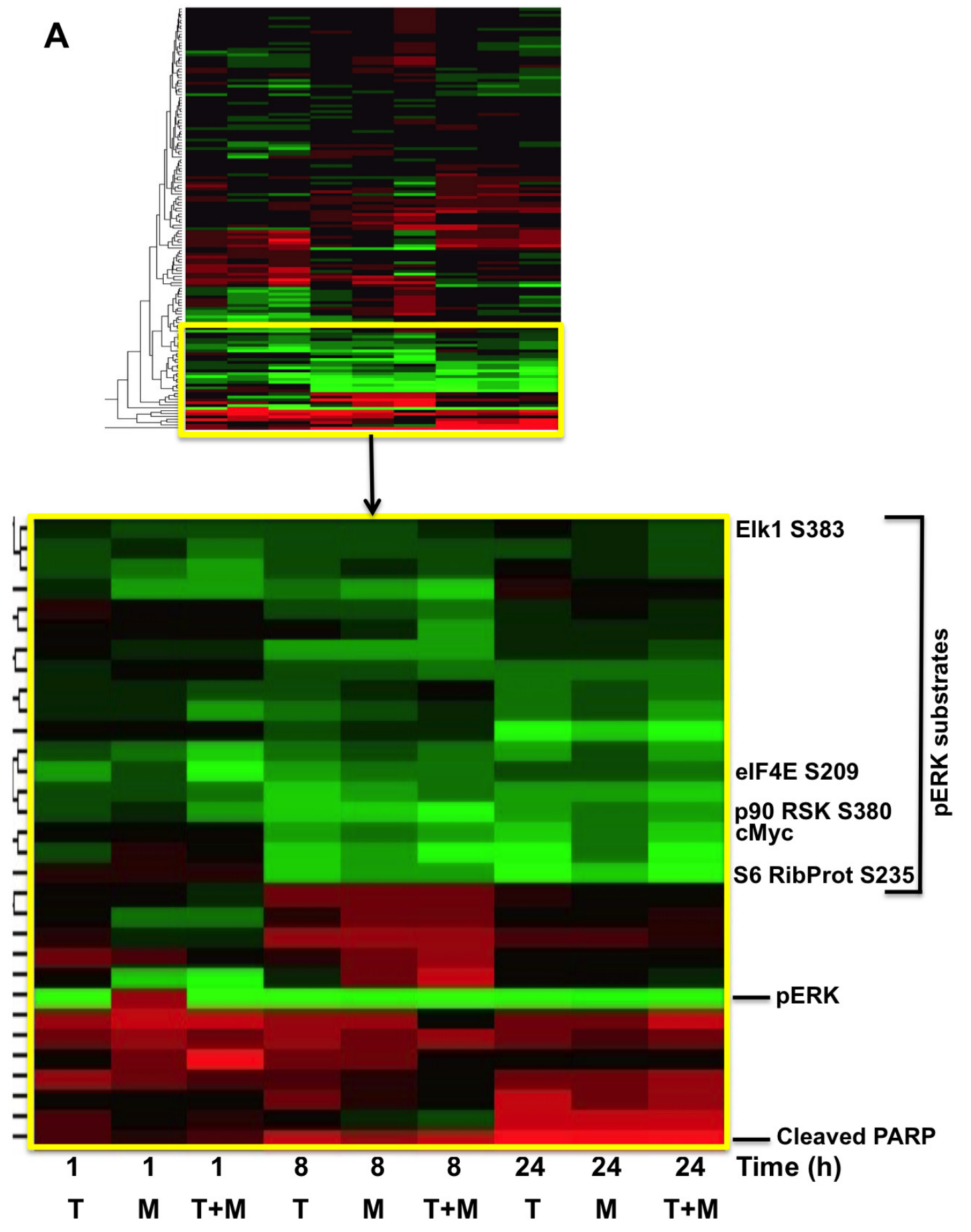


B

MEK

P-MEK

$\begin{array}{llllllllllllllllll}\text { Time (h) } 0 & 0.2 & 0.5 & 1 & 2 & 4 & 8 & 24 & 48 & 0 & 0.2 & 0.5 & 1 & 2 & 4 & 8 & 24 & 48\end{array}$ $\mathrm{ND}-\ldots-\ldots-\ldots-\ldots$

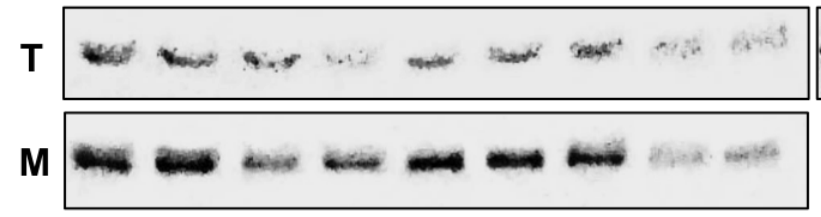

$-\infty-\ldots$

$T+M-\infty \ldots \omega \infty \omega \infty$

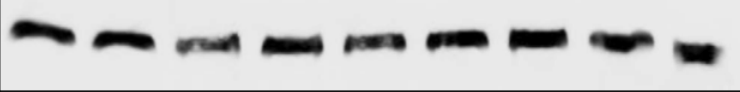

ins

C ERK

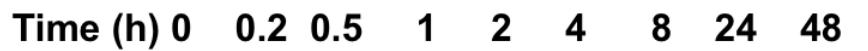
ND $--\infty-\infty-\infty$

$\mathrm{T}$

$\mathbf{M}$

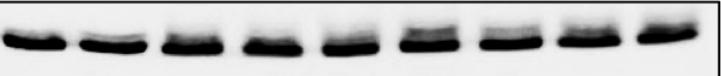

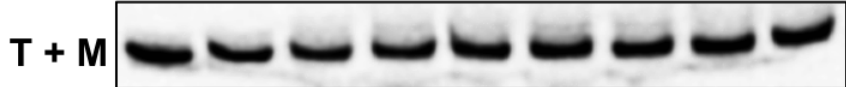

$\begin{array}{ccccccccc}0 & 0.2 & 0.5 & 1 & 2 & 4 & 8 & 24 & 48 \\ - & - & - & - & - & - & - & - & -\end{array}$
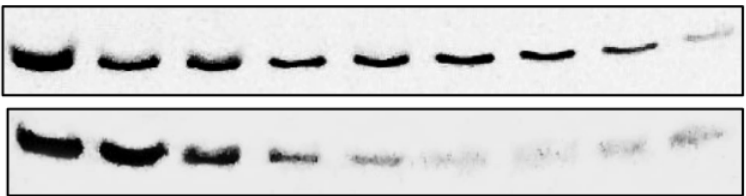

D

BCL2

BCLXL

$\begin{array}{llllllllllllllllll}\text { Time (h) } 0 & 0.2 & 0.5 & 1 & 2 & 4 & 8 & 24 & 48 & 0 & 0.2 & 0.5 & 1 & 2 & 4 & 8 & 24 & 48\end{array}$

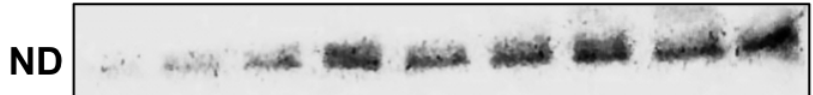

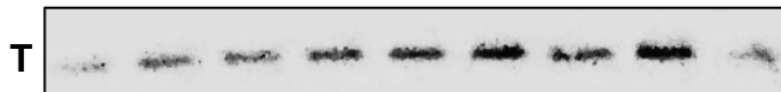

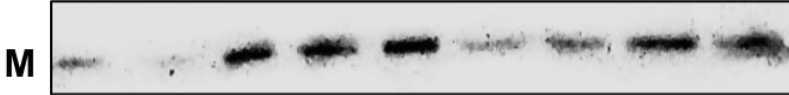

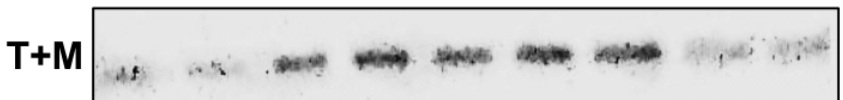
$\sim \sim-\sim-\sim-\cdots$
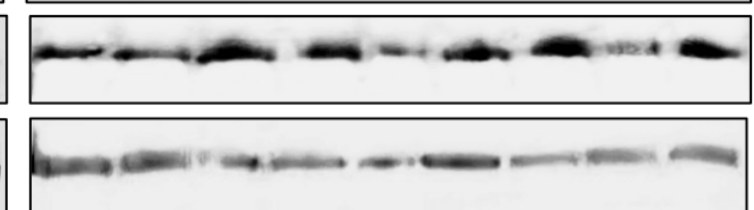

E

pBAD (direct ERK target)

Cleaved PARP

$\begin{array}{lllllllllllllllllll}\text { Time (h) } 0 & 0.2 & 0.5 & 1 & 2 & 4 & 8 & 24 & 48 & 0 & 0.2 & 0.5 & 1 & 2 & 4 & 8 & 24 & 48\end{array}$
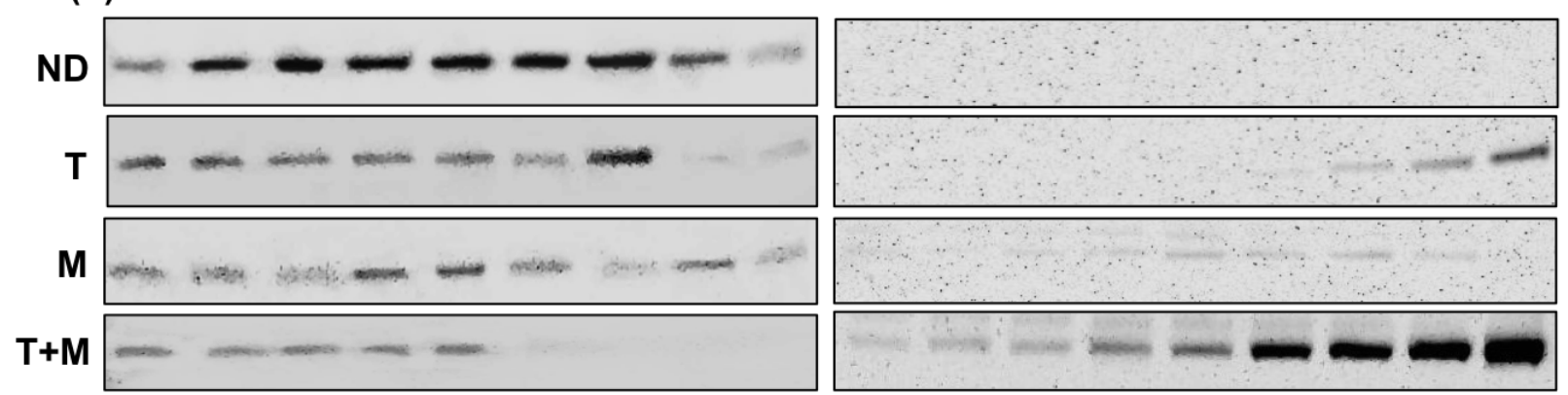


\section{BUL cells}

F
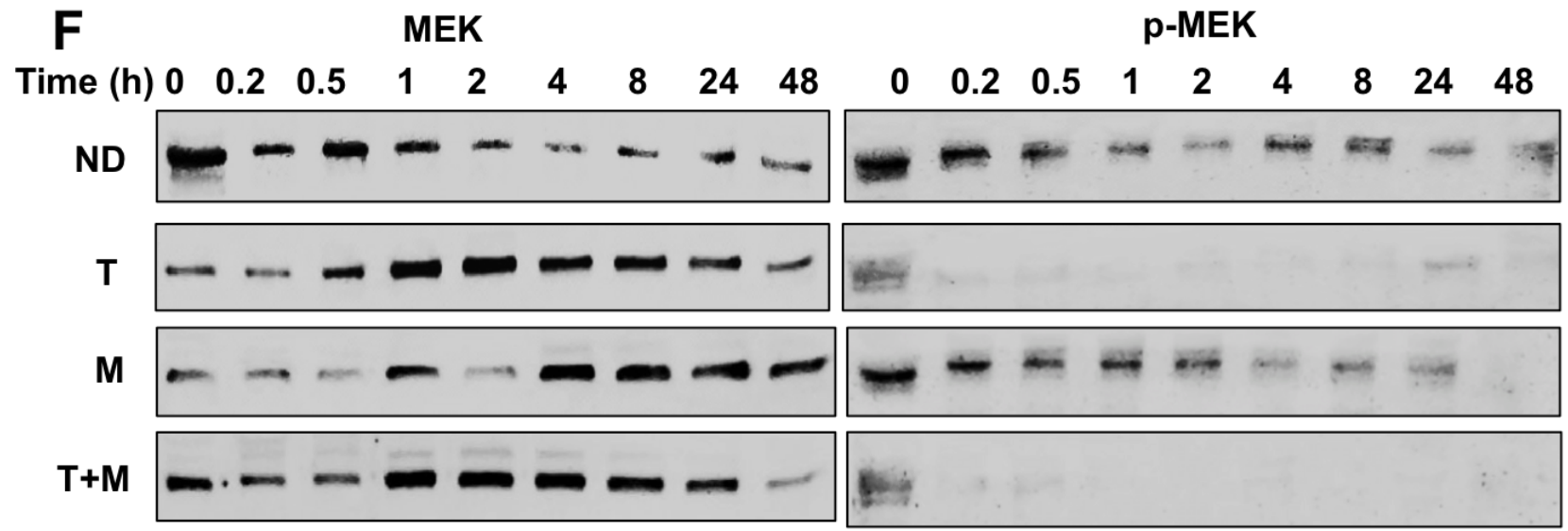

G

ERK
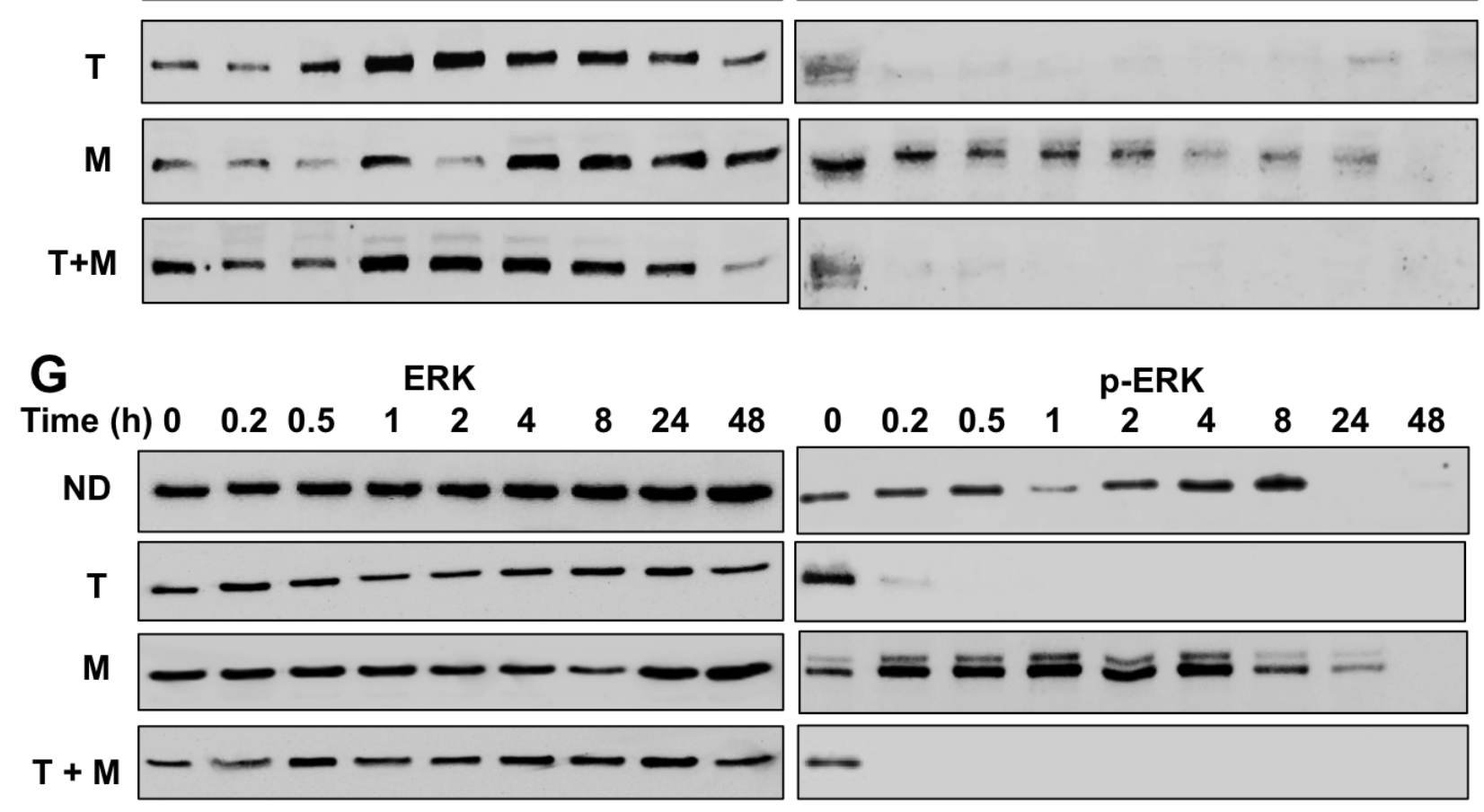

H

pBAD (direct ERK target)

\section{Cleaved PARP}

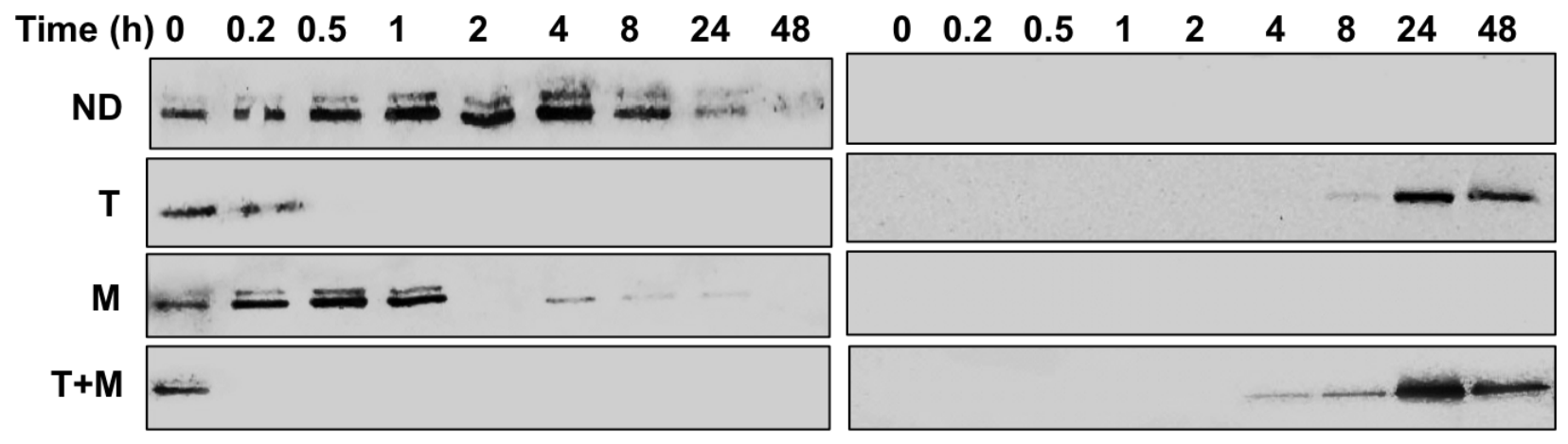

Figure 5: RPPA and immunoblot analyses reveal suppression of the MAPK pathway, including ERK and its downstream substrates in melanoma cells exposed to MBZ+trametinib. A. BAK cells were exposed to $100 \mathrm{nM}$ of MBZ, trametinib, or a combination of the two. Cell extracts derived at indicated times were subjected to RPPA analysis, and unsupervised hierarchal clustering was used to generate a heat map as described in Materials and Methods. B.-E. Immunoblot analyses show marked attenuation of pMEK, pERK, as well as pBAD, coincident with increased levels of cleaved PARP, in melanoma cells exposed to MBZ+trametinib. BAK cells were treated with $100 \mathrm{nM}$ of MBZ, trametinib, or a combination of the two for the indicated times; cell extracts derived and subjected to immunoblot analysis with antibodies specific for total MEK1/2, phospho MEK 1/2 (B), total ERK1/2, phospho-ERK1/2 (C), BCL2, BCLXL (D), phospho-BAD and cleaved PARP (E). All immunoblots were then reprobed with GAPDH as loading control (Supplementary Figures 3-4). 
cleaved PARP (Figure 5E), which is consistent with results observed by RPPA analysis (Figure $5 A$; Supplementary Figure $2 B$ ).

The immunoblot experiments were repeated with an additional melanoma cell line BUL, which harbors the same NRAS ${ }^{\text {Q61K }}$ mutation. Similar to BAK, BUL cells also exhibit a marked attenuation of ERK and MEK phosphorylation within 30 min of $\mathrm{T}+\mathrm{MBZ}$ exposure, coincident with a loss of BAD phosphorylation and increased cleavage of PARP (Figure 5F-5H), demonstrating that both mutant NRAS cells respond strongly to the combination of these two drugs. Taken together, a proposed model for suppression of melanoma growth by MBZ+trametinib is shown in Figure 6.

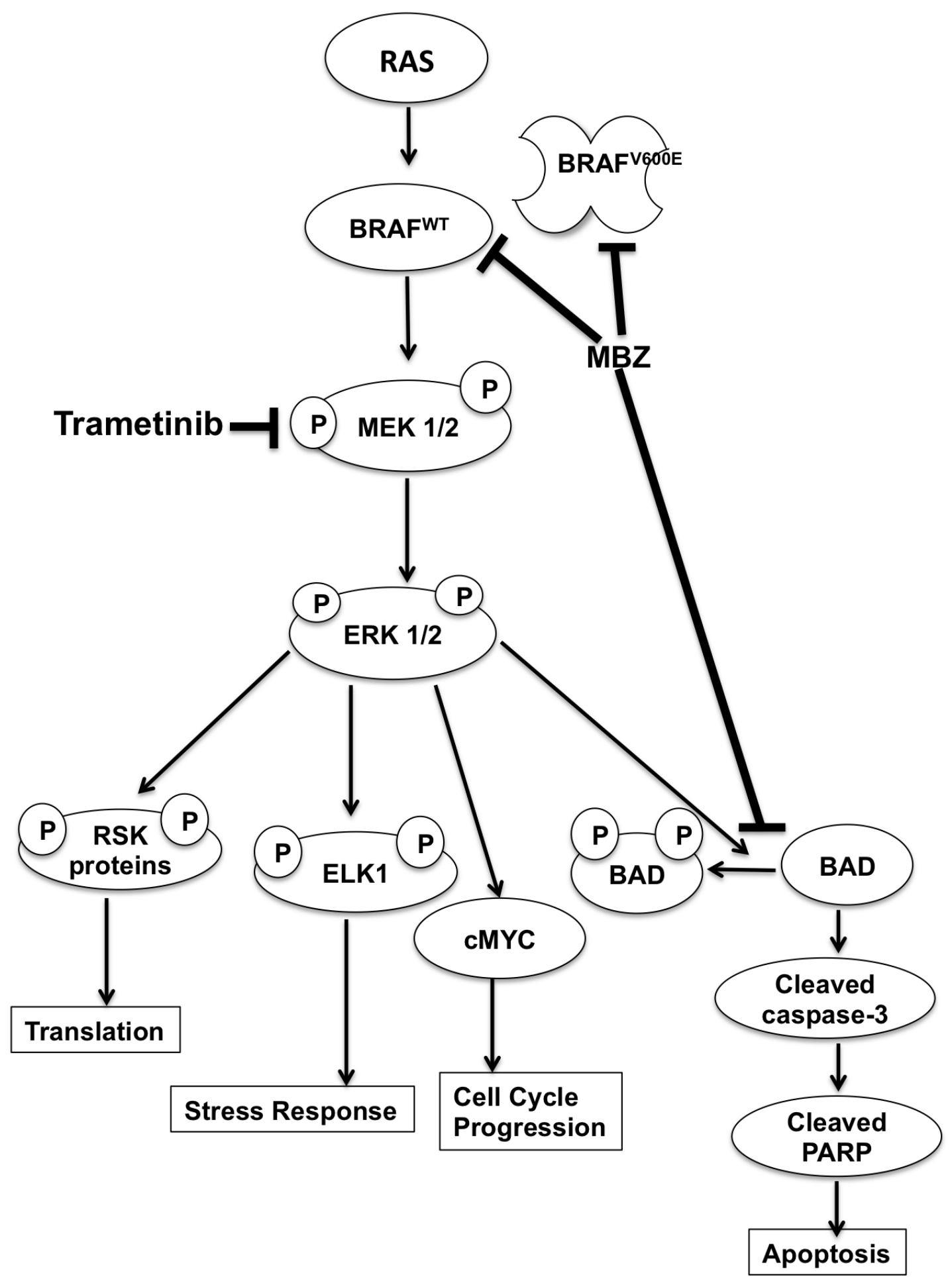

Figure 6: Model of MBZ+trametinib mechanism of action in melanoma cells. 


\section{DISCUSSION}

Our previous results [12] showed that MBZ interacts with VEGFR in silico, which was subsequently corroborated by other investigators who showed that MBZ interacts with additional kinases in vitro, including BCR-ABL and BRAF $[13,16]$. In the current study, we expanded these findings using TMFS to show the nature of the interactions between MBZ and VEGFR, and with $\mathrm{BRAF}^{\mathrm{WT}}$ or BRAF ${ }^{\mathrm{V} 600 \mathrm{E}}$. We also now report the novel finding that MBZ binds both the active and inactive forms of these BRAF proteins. The current study focuses primarily on NRAS ${ }^{\mathrm{mut}} / \mathrm{BRAF}^{\mathrm{WT}}$ melanoma cells, which account for about $21 \%$ of all melanoma cases [28-31]. These have been particularly recalcitrant to treatment, with overall survival times that are shorter than those of patients with melanoma harboring BRAF mutations, and do not respond to BRAFV600 inhibitors such as vemurafinib and dabrafenib. In fact these inhibitors actually enhance growth of NRAS ${ }^{\mathrm{mu}} / \mathrm{BRAF}^{\mathrm{WT}}$ tumors by paradoxically further activating the MAPK pathway through induced conformational changes in wild type RAF isoforms, inducing dimerization, membrane localization, and activation by RAS [32]. The advantages of MBZ are that 1) it interacts with both the active and inactive forms of BRAF, 2) it binds wild type or mutant BRAF with almost equal affinities, and 3 ) it has very low affinity for CRAF, and therefore would not be expected to stimulate tumor growth.

Consistent with our TMFS and kinase assays, MBZ is toxic to patient-derived melanoma cells harboring either WT or mutant BRAF in the presence of trametinib. Further, MBZ+trametinib strongly suppressed the growth of $\mathrm{BRAF}^{\mathrm{WT}} / \mathrm{NRAS}^{\mathrm{Q} 61 \mathrm{~K}}$ melanoma xenografts, and dramatically inhibited ERK1/2 phosphorylation within 10 $\mathrm{min}$. This also blocked phosphorylation of its downstream targets resulting in suppression of proliferation, inhibition of autophagy, and induction of apoptosis; at least in part via suppression of ERK-mediated phosphorylation of BAD, ELK1, decreased expression of Beclin and LC3B, and decreased BCL2 levels. In light of the relative nontoxicity of MBZ, we propose that the MBZ-trametinib combination is a compelling candidate as a therapeutic for patients with metastatic NRAS mutant melanoma.

Previous studies showed the efficacy of MBZ in melanoma cell culture and preclinical mouse models [14, $22,23]$. However, in our mouse xenograft model, MBZ did not work as a monotherapy, but did strongly enhance the effects of trametinib (Figure 4). This might be due to the difference in mutation profiles in our study in which BAK NRAS ${ }^{\mathrm{Q} 61 \mathrm{~K}} / \mathrm{BRAF}^{\mathrm{WT}}$ primary human melanoma cells were used rather than the long-term culture M14 melanoma cells harboring a $\mathrm{NRAS}^{\mathrm{WT}} / \mathrm{BRAF}^{\mathrm{V} 600 \mathrm{E}}$ mutation profile in the previous study. Consistent with the previous study showing BCL2 down-regulation in cells treated with MBZ, our results likewise demonstrate that
$\mathrm{MBZ}+$ trametinib reduces BCL2 levels, as well as BAD S112 phosphorylation, both of which can be explained by the inhibition of MEK/ERK by MBZ+trametinib [26, 27], although some contribution by a lower affinity MBZtubulin interaction cannot be ruled out. Additionally, we observe inhibition of phospho ELK1 S383 at all time points and treatments, a mechanism previously shown to mediate sorafenib-induced endometrial carcinoma apoptosis by lowering MCL1 levels [33] (Figure 5A). MBZ-induced increase in G2 levels was noted (Figure $3 E, 3 J$ ), consistent with previous findings in human lung cancer cells, $[9,10]$. Other differences in the response to MBZ observed previously may be due to the differences in melanoma cell lines, although it should be pointed out that the BAK cells used in the current study harbor a difficult to treat mutation profile.

An important question is whether MBZ and trametinib can reach sufficient concentrations in patients to exert the anti-tumor effects observed in our current cell culture and mouse xenograft studies. One challenge is the relatively poor absorption of MBZ through the gut, which has been unnecessary for the treatment of nematode and cestode parasites resident in the human digestive tract, where it is believed to function by binding tubulin with an apparent binding affinity of $19 \mathrm{nM}$, but has a much lower affinity for human tubulin ( $\mu \mathrm{M}$ range; [6]. However, our results suggest that a major target for MBZ is in fact BRAF, with a higher affinity (Figure 1C) that might be obtained in patients. Oral MBZ can reach peak serum concentrations similar to those used in our current study. For example, in patients treated with chronic MBZ for hydatid disease, a dose of $10 \mathrm{mg} / \mathrm{kg} /$ day resulted in a mean peak plasma level of $470 \mathrm{nM}$, with some variability between patients (0.34-1.69 $\mu \mathrm{M})$ [34], matching half the dose (or 6X the BSA-adjusted dose; Materials and Methods) administered to the mice in this study (40 mg/ $\mathrm{kg} / \mathrm{qad}$; Figure 4), and a plasma concentration equivalent to an IC80 in our cultured NRAS cells (Figure 2A). We are currently testing different formulations of the drug to achieve higher bioavailability and plasma concentrations. MBZ significantly enhanced efficacy of trametinib at 0.1 $\mathrm{mg} / \mathrm{kg} /$ day (LDT) or $3 \mathrm{mg} / \mathrm{kg} /$ day (HDT). Our trametinib doses/[mouse BSA] bracket those prescribed for patients (from $1 / 4-$ to 7 -fold), and our MBZ doses are similar to those used in patients (Materials and Methods) and have been used in previous animal studies [22]. In any case, the trametinib dose-dependent reduction in growth of the MBZ-trametinib treatment groups is promising considering the difficulty treating NRAS tumors, and may work in concert with other drugs. We have collaborated with colleagues at Johns Hopkins who have identified a formulation of MBZ with significantly greater oral absorption. We have also developed an investigatorinitiated Phase IA/B clinical trial using this new formulation of MBZ in combination with a MEK inhibitor for patients with NRAS mutant melanoma that is currently 
under review at a major pharmaceutical company. Based on the encouraging synergy, and likely tolerability of this combination, we are hopeful that the trial will be approved enabling us to formally test this combination in patients.

\section{MATERIALS AND METHODS}

\section{Proteochemometric methods}

A novel rapid computational Proteochemometric method called "Train, Match, Fit, Streamline" (TMFS) was used to map new drug-target interaction space and predict new uses as described [12]. The TMFS method combines shape, topology and chemical signatures, including docking score and functional contact points of the ligand, to predict potential drug-target interactions with remarkable accuracy.

\section{Establishment and characterization of primary human melanoma cell lines}

Human melanoma cell lines were established from fresh metastatic tumor tissues of consenting patients. Tumors were analyzed for mutations in CKIT, BRAF, and $N R A S$ by next generation sequencing. Single cell suspensions were prepared from freshly resected tumor tissue specimens by mechanical mincing; no enzymatic dissociation was used. Viable tumor cells were cultured in Iscove's Modified Dulbecco's Medium (IMDM) supplemented with 10\% fetal bovine serum (FBS) and antibiotics. After overnight incubation at $37^{\circ} \mathrm{C}$ in $5 \%$ $\mathrm{CO}_{2}$, floating debris was discarded and fresh complete medium was added. Cultures were fed 2-3 times per week, replacing half of the spent medium. Melanoma cell lines were split when near confluence and sub-cultured at $4 \times 10^{4}$ viable cells per $\mathrm{cm}^{2}$ surface area in flasks. Cultures were shown to be free of mycoplasma contamination using the MycoProbe ${ }^{\mathrm{TM}}$ mycoplasma detection kit (R\&D Systems, Minneapolis, MN, USA). To ensure that cultured cell lines were melanoma cells, each cell line was stained and analyzed by flow cytometry for melanomaspecific antigens MART-1, gp100, TRP75, or melanomaassociated chondroitin sulfate proteoglycan. All cell lines were early passages of less than 20 .

\section{Cell culture}

Patient-derived melanoma cell lines (BAK and BUL) with the same $\mathrm{BRAF}^{\mathrm{WT}} / \mathrm{NRAS}^{\mathrm{Q} 61 \mathrm{~K}}$ mutation signature, as well as a melanoma cell line (STU) harboring a $\mathrm{BRAF}^{\mathrm{V} 600 \mathrm{~K}} / \mathrm{NRAS}^{\mathrm{WT}}$ mutation were cultured in IMDM with $10 \% \mathrm{FBS}$ and $1 \%$ penicillin/streptomycin in a $5 \%$ $\mathrm{CO}_{2}$ incubator at $37^{\circ} \mathrm{C}$. Cell growth was monitored daily and expanded to obtain sufficient cell numbers for subsequent experiments. Mutation signatures of cell lines were confirmed by PCR and sequencing.

\section{Drug toxicity assays}

MBZ, dabrafenib, and trametinib were purchased from Sigma-Aldrich and ActiveBiochem, respectively. 5 x $10^{3}$ viable cells per well were plated in 96-well dishes and allowed to recover for $12 \mathrm{~h}$ prior to drug treatment. Cells in triplicate wells were treated for up to $72 \mathrm{~h}$ (based on initial time course experiments showing maximal effects at that time point) with different concentrations of trametinib, dabrafenib, or MBZ alone, or a combination of MBZ and trametinib. Negative controls were exposed to vehicle DMSO in the same volumes. Cell viability was assessed by an XTT assay, according to a manufacturer's specifications (Biotium Inc). Reduced XTT was measured by absorbance at $490 \mathrm{~nm}$ on a PerkinElmer Victor3 plate reader. Cells exposed to detergent served as a positive control.

\section{Cell cycle analysis}

Cells were collected, fixed in ethanol, stained with propidium iodide (PI) to determine DNA content, and analyzed by flow cytometry (FACStar PLus; BD BioSciences, San Jose, CA).

\section{Mouse xenografting}

All animal experiments were performed in accordance with the guidelines and approval of Georgetown University Animal Care and Use Committee. Athymic 6-week old male mice (Taconic) were acclimated to the Division of Comparative Medicine at Georgetown University a week prior to xenografting. $3 \times 10^{6}$ melanoma cells were resuspended in Matrigel and injected subcutaneously into the hind flanks of athymic mice using a 22-gauge needle. Tumor growth was measured with calipers, and drug treatment started when tumor volumes reached $100 \mathrm{~mm}^{3}$, after which mice were monitored daily for drug efficacy, as well for adverse effects, including weight and behavior. Drugs were administered by oral gavage. Each testing group contained three to five mice in each of four experiments. Each tumor from each treatment group was measured on indicated days, and all tumor sizes were then normalized to their size at day 0 of drug treatment. All data from all four experiments was then combined for statistical analysis, to compare every mouse from each treatment group. The total mice for all experiments included vehicle control $(n=13)$, low-dose $\mathrm{T}(n=12)$, MBZ $(n=12)$, high-dose T $(n=12)$, lowdose trametinib $+\mathrm{MBZ}(n=12)$, and high-dose trametinib 
$+\mathrm{MBZ}(n=15)$. The results were expressed as the mean $( \pm \mathrm{SD})$ of tumor volume in each group. After five weeks, mice were euthanized; tumors extracts were derived for immunoblot analysis.

\section{Dosing}

Trametinib doses were calculated relative to doses prescribed for patients, based on weight and body surface area for mouse and human, using the surface area to weight ratios $\left(\mathrm{m}^{2} / \mathrm{kg}\right)$ described for mouse $(.02 \mathrm{~kg} / 0.0066$ $\left.\mathrm{m}^{2}=3.0\right)$ and human $\left(60 \mathrm{~kg} / 1.6 \mathrm{~m}^{2}=37\right)[35]$. This yields a similar constant to that calculated by Mosteller for humans: BSA $\left(\mathrm{m}^{2}\right)=\left[\mathrm{SQRT}(\mathrm{H}(\mathrm{cm}) x \mathrm{~W}(\mathrm{~kg})]^{* 60}\right.$ [36] Our mouse "low dose" trametinib $(0.1 \mathrm{mg} / \mathrm{kg})$, adjusting for surface area $=3 / 37^{*}(0.1 \mathrm{mg} / \mathrm{kg})=0.008 \mathrm{mg} / \mathrm{kg}$, is therefore equivalent to a human dose of $0.008 \mathrm{mg} / \mathrm{kg} * 60$ $\mathrm{kg}$ (average body mass globally) $=0.48 \mathrm{mg} /$ person/day . For high dose trametinib, this is equivalent to $14.4 \mathrm{mg} /$ person/day. By comparison, the dose for patients is $2 \mathrm{mg}$ PO qDay. For MBZ, our dose of $40 \mathrm{mg} / \mathrm{kg}$, adjusted for surface area constants $3 / 37$ (mouse/human), is equivalent to $3 / 37 *(40 \mathrm{mg} / \mathrm{kg}) * 60 \mathrm{~kg} /$ person $=195 \mathrm{mg}$. Вy comparison, patient doses range from $100 \mathrm{mg}$ one time (pinworms) to $200 \mathrm{mg} / \mathrm{kg}$ per day for 12 weeks (hyatid disease in children) including doses up to $6 \mathrm{~g}$ per day [5]. In summary, our trametinib doses/[mouse BSA] bracket those prescribed for patients (from $1 / 4$ - to 7-fold), and our MBZ doses are much lower than those that have been safely used in patients $[5,37]$, and similar to those used in other preclinical studies $[22,23]$.

\section{Immunoblot analysis}

SDS-PAGE and transfer of separated proteins to nitrocellulose membranes were performed according to standard procedures. Membranes were stained with Ponceau S $(0.1 \%)$ to verify equal loading and transfer of proteins, and then incubated with antibodies specific for pERK1/2 T202/Y204, pMEK1/2 S217/221, pBAD S-112, total BAD, total ERK1/2, total MEK1/2, BCL2 (Santa Cruz Biotech), BCLXL (Santa Cruz Biotech), cleaved PARP (Cell Signaling), or GAPDH (Abcam; loading control). Immune complexes were detected by incubation with appropriate horseradish peroxidase-conjugated antibodies to mouse or rabbit IgG (1:3000) and enhanced chemiluminescence (Pierce, Rockford, IL).

\section{Fluorometric caspase-3 activity}

Cytosolic extracts, derived from pooled floating and attached cells, were subjected to fluorometric caspase-3 activity assays using fluorescent tetrapeptide substrate specific for caspases-3 (Ac-DEVD-aminomethylcoumarin
(AMC, Enzo Life Sciences, Ann Arbor, MI) as previously described [38]. Free AMC, generated as a result of cleavage of the aspartate-AMC bond, was monitored over 30 min with a Wallac Victor ${ }^{3}$ fluorometer (Perkin-Elmer, Waltham, MA) at excitation and emission wavelengths of 360 and $460 \mathrm{~nm}$, respectively. The emission from each sample was plotted against time, and linear regression analysis of the initial velocity (slope) for each curve yielded the activity.

\section{Reverse-phase protein arrays}

Cell lysates were analyzed by reverse-phase protein array (RPPA) [39]. Samples were diluted to $0.5 \mathrm{mg}$ / $\mathrm{mL}$ and dilutions printed on slides in triplicate. Slides were immunostained with 137 different antibodies specific for total proteins, or phosphorylated or cleaved products. Analytes measured were chosen based on their "actionability" (e.g. were known drug targets for FDAapproved drugs, drugs in clinical trials, or targets of other commercially-available compounds), as well as for and their known involvement in tumorigenesis and cancer biology and components in key signaling pathways that control cell growth, motility, inflammation, autophagy, survival, differentiation and apoptosis. All antibodies have been pre-validated for specificity by immunoblot analysis. Intensity values were normalized to that of total protein for each sample stained with Sypro Ruby (Invitrogen). Unsupervised cluster analysis http://www.hiv.lanl. gov/content/sequence/HEATMAP/heatmap.html was performed for all proteins in the RPPA using the standard bootstrap method.

\section{Statistical analysis}

The results shown are based on a single experiment in triplicate, and repeated in three independent experiments with essentially the same results. Data from triplicates of treatment groups were compared using Student's t-test or 2-way ANOVA (multiple comparisons) for significance, and $p$ values of $<0.05$ were considered statistically significant. For tumor sizes, the rate-based T/C (tumor/ control) test of significance was used as described, using the author's template [40]. The results are representative of 3 independent experiments with reproducible results. For determining synergism, the combination index $(\tau)$ was calculated from single dose-response curves and combination experiments as $\tau=x_{\mathrm{A}} / \mathrm{X}_{\mathrm{A}}+x_{\mathrm{B}} / \mathrm{X}_{\mathrm{B}}$, in which, for a given cytotoxic effect, $x_{\mathrm{A}}$ and $x_{\mathrm{B}}$ are the concentrations of drugs $A$ and $B$ in the combination, and $\mathrm{X}_{\mathrm{A}}$ and $\mathrm{X}_{\mathrm{B}}$ are the concentrations of drugs $A$ and $B$ that achieve the same cytotoxic effect when given alone [41]. A $\tau$ value of 1 indicates additivity, $\tau$ less than 1 indicates synergy, and $\tau$ greater than 1 indicates antagonism. 


\section{ACKNOWLEDGMENTS}

The authors would like to thank Zhixin Hui, Qing Qin, and Dr. Patricia Foley (DVM) for help with mouse gavage treatments, and Sushma Sunil, Manish Moghe, Vikas Soni, Drs. Rena Shimizu and Maria Fairbanks for technical assistance with the in vitro experiments.

\section{CONFLICTS OF INTEREST}

The authors declare no conflicts of interest.

\section{GRANT SUPPORT}

These studies were funded in part by Developmental funds from the Lombardi Comprehensive Cancer Center and a MedStar-Georgetown partnership grant to DR. SD and SB were supported by NIH-R01 CA170653 and DOD grant CA140882 (SB, DR, SD).

\section{REFERENCES}

1. Lovly CM, Shaw AT. Molecular pathways: resistance to kinase inhibitors and implications for therapeutic strategies. Clin Cancer Res. 2014; 20: 2249-56. doi: 10.1158/10780432.CCR-13-1610.

2. Kollmannsberger C, Bjarnason G, Burnett P, Creel P, Davis M, Dawson N, Feldman D, George S, Hershman J, Lechner T, Potter A, Raymond E, Treister N, et al. Sunitinib in metastatic renal cell carcinoma: recommendations for management of noncardiovascular toxicities. Oncologist. 2011; 16: 543-53. doi: 10.1634/theoncologist.2010-0263.

3. Paul SM, Mytelka DS, Dunwiddie CT, Persinger CC, Munos BH, Lindborg SR, Schacht AL. How to improve R\&D productivity: the pharmaceutical industry's grand challenge. Nat Rev Drug Discov. 2010; 9: 203-14. doi: 10.1038/nrd3078.

4. Banerjee D, Mandal A, Prakash O. Mebendazole (R17,635): a new anthelmintic in the development of hookworms. Trans R Soc Trop Med Hyg. 1971; 65: 685-6.

5. Messaritakis J, Psychou P, Nicolaidou P, Karpathios T, Syriopoulou B, Fretzayas A, Krikos F, Matsaniotis N. High mebendazole doses in pulmonary and hepatic hydatid disease. Arch Dis Child. 1991; 66: 532-3.

6. Friedman PA, Platzer EG. Interaction of anthelmintic benzimidazoles with Ascaris suum embryonic tubulin. Biochim Biophys Acta. 1980; 630: 271-8.

7. Laclette JP, Guerra G, Zetina C. Inhibition of tubulin polymerization by mebendazole. Biochem Biophys Res Commun. 1980; 92: 417-23.

8. Argyriou AA, Kyritsis AP, Makatsoris T, Kalofonos HP. Chemotherapy-induced peripheral neuropathy in adults: a comprehensive update of the literature. Cancer Manag Res. 2014; 6: 135-47. doi: 10.2147/CMAR.S44261.
9. Mukhopadhyay T, Sasaki J, Ramesh R, Roth JA. Mebendazole elicits a potent antitumor effect on human cancer cell lines both in vitro and in vivo. Clin Cancer Res. 2002; 8: 2963-9.

10. Sasaki J, Ramesh R, Chada S, Gomyo Y, Roth JA, Mukhopadhyay T. The anthelmintic drug mebendazole induces mitotic arrest and apoptosis by depolymerizing tubulin in non-small cell lung cancer cells. Mol Cancer Ther. 2002; 1: 1201-9.

11. Issa NT, Peters OJ, Byers SW, Dakshanamurthy S. RepurposeVS: A Drug Repurposing-Focused Computational Method for Accurate Drug-Target Signature Predictions. Comb Chem High Throughput Screen. 2015; 18: 784-94.

12. Dakshanamurthy S, Issa NT, Assefnia S, Seshasayee A, Peters OJ, Madhavan S, Uren A, Brown ML, Byers SW. Predicting new indications for approved drugs using a proteochemometric method. J Med Chem. 2012; 55: 683248. doi: 10.1021/jm300576q.

13. Nygren P, Fryknas M, Agerup B, Larsson R. Repositioning of the anthelmintic drug mebendazole for the treatment for colon cancer. J Cancer Res Clin Oncol. 2013; 139: 2133-40. doi: 10.1007/s00432-013-1539-5.

14. Doudican N, Rodriguez A, Osman I, Orlow SJ. Mebendazole induces apoptosis via Bcl-2 inactivation in chemoresistant melanoma cells. Mol Cancer Res. 2008; 6: 1308-15. doi: 10.1158/1541-7786.MCR-07-2159.

15. Dobrosotskaya IY, Hammer GD, Schteingart DE, Maturen KE, Worden FP. Mebendazole monotherapy and long-term disease control in metastatic adrenocortical carcinoma. Endocr Pract. 2011; 17: e59-62. doi: 10.4158/EP10390.CR.

16. Nygren P, Larsson R. Drug repositioning from bench to bedside: tumour remission by the antihelmintic drug mebendazole in refractory metastatic colon cancer. Acta Oncol. 2014; 53: 427-8. doi: 10.3109/0284186X.2013.844359.

17. Pantziarka P, Bouche G, Meheus L, Sukhatme V, Sukhatme VP. Repurposing Drugs in Oncology (ReDO)-mebendazole as an anti-cancer agent. Ecancermedicalscience. 2014; 8: 443. doi: 10.3332/ecancer.2014.443.

18. Wan PT, Garnett MJ, Roe SM, Lee S, Niculescu-Duvaz D, Good VM, Jones CM, Marshall CJ, Springer CJ, Barford D, Marais R, Cancer Genome P. Mechanism of activation of the RAF-ERK signaling pathway by oncogenic mutations of B-RAF. Cell. 2004; 116: 855-67.

19. Tseng JR, Stuart D, Aardalen K, Kaplan A, Aziz N, Hughes NP, Gambhir SS. Use of DNA microarray and small animal positron emission tomography in preclinical drug evaluation of RAF265, a novel B-Raf/VEGFR-2 inhibitor. Neoplasia. 2011; 13: 266-75.

20. Bollag G, Tsai J, Zhang J, Zhang C, Ibrahim P, Nolop K, Hirth P. Vemurafenib: the first drug approved for BRAFmutant cancer. Nat Rev Drug Discov. 2012; 11: 873-86. doi: $10.1038 / \operatorname{nrd} 3847$. 
21. Niculescu-Duvaz D, Niculescu-Duvaz I, Suijkerbuijk BM, Menard D, Zambon A, Nourry A, Davies L, Manne HA, Friedlos F, Ogilvie L, Hedley D, Takle AK, Wilson DM, et al. Novel tricyclic pyrazole BRAF inhibitors with imidazole or furan central scaffolds. Bioorg Med Chem. 2010; 18: 6934-52. doi: 10.1016/j.bmc.2010.06.031.

22. Doudican NA, Byron SA, Pollock PM, Orlow SJ. XIAP downregulation accompanies mebendazole growth inhibition in melanoma xenografts. Anticancer Drugs. 2013; 24: 181-8. doi: 10.1097/CAD.0b013e32835a43f1.

23. Bai RY, Staedtke V, Wanjiku T, Rudek MA, Joshi A, Gallia GL, Riggins GJ. Brain Penetration and Efficacy of Different Mebendazole Polymorphs in a Mouse Brain Tumor Model. Clin Cancer Res. 2015; 21: 3462-70. doi: 10.1158/10780432.CCR-14-2681.

24. Kim JH, Hong SK, Wu PK, Richards AL, Jackson WT, Park JI. Raf/MEK/ERK can regulate cellular levels of LC3B and SQSTM1/p62 at expression levels. Exp Cell Res. 2014; 327: 340-52. doi: 10.1016/j.yexcr.2014.08.001.

25. Jang GH, Lee M. BH3-mimetic gossypol-induced autophagic cell death in mutant BRAF melanoma cells with high expression of p21Cip(1).). Life Sci. 2014; 102: 41-8. doi: 10.1016/j.lfs.2014.02.036.

26. Boucher MJ, Morisset J, Vachon PH, Reed JC, Laine J, Rivard N. MEK/ERK signaling pathway regulates the expression of $\mathrm{Bcl}-2, \mathrm{Bcl}-\mathrm{X}(\mathrm{L})$, and $\mathrm{Mcl}-1$ and promotes survival of human pancreatic cancer cells. J Cell Biochem. 2000; 79: 355-69.

27. Hayakawa J, Ohmichi M, Kurachi H, Kanda Y, Hisamoto K, Nishio Y, Adachi K, Tasaka K, Kanzaki T, Murata Y. Inhibition of BAD phosphorylation either at serine 112 via extracellular signal-regulated protein kinase cascade or at serine 136 via Akt cascade sensitizes human ovarian cancer cells to cisplatin. Cancer Res. 2000; 60: 5988-94.

28. Platz A, Egyhazi S, Ringborg U, Hansson J. Human cutaneous melanoma; a review of NRAS and BRAF mutation frequencies in relation to histogenetic subclass and body site. Mol Oncol. 2008; 1: 395-405. doi: 10.1016/j. molonc.2007.12.003.

29. Ball NJ, Yohn JJ, Morelli JG, Norris DA, Golitz LE, Hoeffler JP. Ras mutations in human melanoma: a marker of malignant progression. J Invest Dermatol. 1994; 102: 285-90.

30. Curtin JA, Fridlyand J, Kageshita T, Patel HN, Busam KJ, Kutzner H, Cho KH, Aiba S, Brocker EB, LeBoit PE, Pinkel D, Bastian BC. Distinct sets of genetic alterations in melanoma. N Engl J Med. 2005; 353: 2135-47. doi: 10.1056/NEJMoa050092.
31. van 't Veer LJ, Burgering BM, Versteeg R, Boot AJ, Ruiter DJ, Osanto S, Schrier PI, Bos JL. N-ras mutations in human cutaneous melanoma from sun-exposed body sites. Mol Cell Biol. 1989; 9: 3114-6.

32. Hatzivassiliou G, Song K, Yen I, Brandhuber BJ, Anderson DJ, Alvarado R, Ludlam MJ, Stokoe D, Gloor SL, Vigers G, Morales T, Aliagas I, Liu B, et al. RAF inhibitors prime wild-type RAF to activate the MAPK pathway and enhance growth. Nature. 2010; 464: 431-5. doi: 10.1038/ nature 08833 .

33. Sun NK, Huang SL, Chang TC, Chao CC. Sorafenib induces endometrial carcinoma apoptosis by inhibiting Elk-1-dependent Mcl-1 transcription and inducing Akt/ GSK3beta-dependent protein degradation. J Cell Biochem. 2013; 114: 1819-31. doi: 10.1002/jcb.24530.

34. Braithwaite PA, Roberts MS, Allan RJ, Watson TR. Clinical pharmacokinetics of high dose mebendazole in patients treated for cystic hydatid disease. Eur J Clin Pharmacol. 1982; 22: 161-9.

35. Freireich EJ, Gehan EA, Rall DP, Schmidt LH, Skipper HE. Quantitative comparison of toxicity of anticancer agents in mouse, rat, hamster, dog, monkey, and man. Cancer Chemother Rep. 1966; 50: 219-44.

36. Mosteller RD. Simplified calculation of body-surface area. N Engl J Med. 1987; 317: 1098. doi: 10.1056/ NEJM198710223171717.

37. Bekhti A, Pirotte J. Cimetidine increases serum mebendazole concentrations. Implications for treatment of hepatic hydatid cysts. Br J Clin Pharmacol. 1987; 24: 390-2.

38. Simbulan-Rosenthal CM, Velena A, Veldman T, Schlegel R, Rosenthal DS. HPV-16 E6/7 immortalization sensitizes human keratinocytes to ultraviolet $\mathrm{B}$ by altering the pathway from caspase-8 to caspase-9-dependent apoptosis. J Biol Chem. 2002; 277: 24709-16.

39. Einspahr JG, Calvert V, Alberts DS, Curiel-Lewandrowski C, Warneke J, Krouse R, Stratton SP, Liotta L, Longo C, Pellacani G, Prasad A, Sagerman P, Bermudez Y, et al. Functional protein pathway activation mapping of the progression of normal skin to squamous cell carcinoma. Cancer Prev Res (Phila). 2012; 5: 403-13. doi: 10.1158/1940-6207.CAPR-11-0427.

40. Hather G, Liu R, Bandi S, Mettetal J, Manfredi M, Shyu WC, Donelan J, Chakravarty A. Growth rate analysis and efficient experimental design for tumor xenograft studies. Cancer Inform. 2014; 13: 65-72. doi: 10.4137/CIN.S13974.

41. Berenbaum MC. Synergy, additivism and antagonism in immunosuppression. A critical review. Clin Exp Immunol. 1977; 28: 1-18. 